\title{
Reliability-based topology optimization of uncertain building systems subject to stochastic excitation
}

\author{
Sarah Bobby ${ }^{b}$, Arthriya Suksuwan ${ }^{\mathrm{a}}$, Seymour M.J. Spence ${ }^{\mathrm{a}, 1, *}$, Ahsan \\ Kareem $^{b}$ \\ ${ }^{a}$ Department of Civil and Environmental Engineering, University of Michigan, Ann \\ Arbor, MI 48109, USA \\ ${ }^{b}$ NatHaz Modeling Laboratory, Department of Civil and Environmental Engineering and \\ Earth Sciences, University of Notre Dame, Notre Dame, IN 46556, USA
}

\begin{abstract}
Topology optimization has traditionally been developed in a deterministic setting, notwithstanding the considerable uncertainties that generally affect both the system as well as the excitation. Therefore, the development of methods that are capable of describing the performance of uncertain systems in a fully probabilistic setting would represent an important step forward. In particular, the ability to consider reliability constraints written in terms of first excursion probabilities posed on systems driven by general stochastic excitation would allow a wide variety of important design scenarios to be modeled. This paper is focused on proposing a simulation-centered reliability-based topology optimization framework to this end. In particular, an approach is developed based on defining, from the argumentation of the simulation process, an optimization sub-problem that not only approximately decouples the probabilistic analysis from the optimization loop, but takes a form that can be extremely efficiently solved. By solving a limited sequence of sub-problems, solutions are found that rigorously meet the first excursion constraints of the original problem. A series of case studies are presented illustrating the potential of the proposed framework.
\end{abstract}

Keywords: Reliability-Based Design, Topology Optimization, Seismic

\footnotetext{
*Corresponding author

Email addresses: sbobby@nd.edu (Sarah Bobby), arthriya@umich.edu (Arthriya Suksuwan), smjs@umich.edu (Seymour M.J. Spence), kareem@nd.edu (Ahsan Kareem)

${ }^{1}$ Tel. +1-734-764-8419, Fax +1-734-764-4292
} 
Engineering, Monte Carlo Simulation, Stochastic Systems

\section{Introduction}

Topology optimization is a powerful conceptual design tool that can be applied to a wide variety of engineering problems. The strength of topology optimization lies in its ability to systematically explore complex design spaces. Within the field of structural engineering, it can be used as a formal approach for the identification of optimal load-resisting systems [1-10]. Notwithstanding the considerable uncertainties that generally affect the system (e.g. dynamic and material properties) and excitation (e.g. earthquakes and wind) of many practical applications, the majority of work on topology optimization has been performed in a deterministic setting. This can lead to systems that exhibit poor performance, or even fail. In order to avoid this, the uncertainties affecting the problem must be rationally treated through, for example, reliability methods. In the case of uncertain systems with fixed topologies, reliability-based optimization methods are relatively well established (for a review see e.g. [11, 12]). The same cannot be said for the topology optimization of uncertain system, where the difficulty in developing such a framework can be traced back to how topology optimization problems are inevitably characterized by large design variable vectors (in the order of thousands) that are necessary for adequately discretizing the design domain. When this is combined with systems driven by general stochastic excitation and subject to constraints on first excursion probabilities, a problem is defined that is not only in terms of a large design variable vector but also of high-dimensional implicit probabilistic integrals. This combination is challenging, as the presence of a large design variable vector significantly complicates the calculation of the sensitivities of the implicit reliability constraints, while making methods based on developing local or global approximations of the reliability constraints computationally intractable. Indeed, the majority of the research on reliability-based topology optimization (RBTO), or more generally topology optimization under uncertainty, has focused on static problems characterized by relatively few sources of uncertainty, as for example, material properties [13-16], applied static loads [13, 14, 17-21], nonstructural mass [18], or geometric uncertainties [16, 17, 19, 22].

Recently, there has been growing interest in developing methods that can include stochastic excitation $[7,8,10,23,24]$. In particular, Chun et al. 
$[23,24]$ have developed a method for deterministic structures subject to stochastic excitation modeled as a Gaussian process using a classic discrete representation method [25]. The approach is based on calculating the instantaneous failure probability, as opposed to the more general but challenging first excursion probability, through first order reliability methods (FORM). The approach is limited by the excitation model and the unavoidable limitations of FORM [26]. In Kareem et al. [8] and Bobby et al. [10] a framework is proposed for the topology optimization of uncertain systems subject to reliability constraints (in terms of fragility), where it was assumed that the excitation was stationary, Gaussian and ergodic, therefore leading to a closed form for the failure probability. This can be limiting, as the stochastic excitation generated by many natural hazards (e.g. earthquakes, but also thunderstorms, hurricanes, and tornadoes [27]) may be non-stationary and/or non-Gaussian. While it is traditional to assume stationarity in order to facilitate calculations, this inevitably introduces a source of model uncertainty during the determination of the system's reliability. The development of optimization frameworks that allow the use of non-stationary stochastic excitation would allow the user to decide the most appropriate reliability model to use for describing the performance of the system.

This paper is focused on the development of such a framework. In particular, a simulation-based RBTO framework is proposed for the conceptual design of uncertain structures subject to stochastic excitation and reliability constraints written in terms of first excursion probabilities.

\section{Reliability-Based Topology Optimization}

\subsection{Design domain}

In order to apply topology optimization to the design of building systems, an appropriate design domain must first be defined. In particular, it is the lateral load-resisting system that is generally designed to withstand external environmental loads and is therefore of interest here. As a consequence, the design domain, i.e. the space in which elements of the lateral loadresisting system are permitted to exist, can be seen as a subset of the external envelope of the building. It is this domain, here indicated as $\Omega$, that will be discretized with finite elements (FE) and then optimized. Because the generally non-designable secondary system (floor and gravity system) is an important and unavoidable source of stiffness for the lateral load-resisting system, it must also be modeled in order to ensure a realistic overall stiffness 
[10]. Therefore, an effective complete FE model of the system can be defined as the superposition of a FE model of the secondary system and a FE model discretizing the design domain, as shown in Fig. 1. If it is also observed that the majority of mass in a typical multistory building can be found at the floor levels [28], it becomes reasonable to consider the mass of the secondary system (including any carried mass) as well as the mass associated with the elements of the design domain concentrated at the floor levels. Because the flexibility of the floor systems must be considered, the mass cannot be lumped at a single node (e.g. the center of mass) per floor as is typical in building design [28]. Instead, the mass must be considered appropriately distributed over the floor area. From a modeling prospective, the distributed mass can be lumped at a number of master nodes belonging to the floor system as illustrated in Fig. 1.

If it is also ensured that the master nodes, i.e. mass nodes, belong to the FE model of the secondary system, for mass dependent loading (i.e. seismic and dynamic loading) the design domain becomes independent of the loading scheme. In other words, the external loads can always find a load path through the floor system to the main lateral load-resisting system. This is an extremely desirable property as it ensures that the resulting topologies are not guided by the choice of the master nodes. This will result in a greater optimality of the final designs while ensuring a realistic load path for mass dependent loads. In this work, the stiffness matrix of the complete system will be indicated as $\mathbf{K}$ while the corresponding mass matrix will be indicated as $\mathbf{M}$.

\subsection{Problem setting}

In order to find optimal topologies in the design domain identified above while considering an uncertain system subject to external stochastic excitation, solutions to the following reliability-based topology optimization must be found:

$$
\begin{aligned}
\min _{\boldsymbol{\rho}} & V(\boldsymbol{\rho})=\sum_{e=1}^{n} \int_{\Omega_{e}} \rho_{e} \mathrm{~d} \Omega \\
\text { s.t. } & \mathrm{P}\left(G_{j}\left(\boldsymbol{\rho}, \mathbf{U}_{s}, \mathbf{U}_{g}, \mathbf{X}\right) \leq 0\right) \leq \mathrm{P}_{0_{j}}, j=1, \ldots, N \\
& 0 \leq \rho_{e} \leq 1
\end{aligned}
$$

where $\boldsymbol{\rho}=\left\{\rho_{1}, \ldots, \rho_{n}\right\}^{T}$ is the element-wise normalized material density design variable vector that is generally related to an independent design variable 


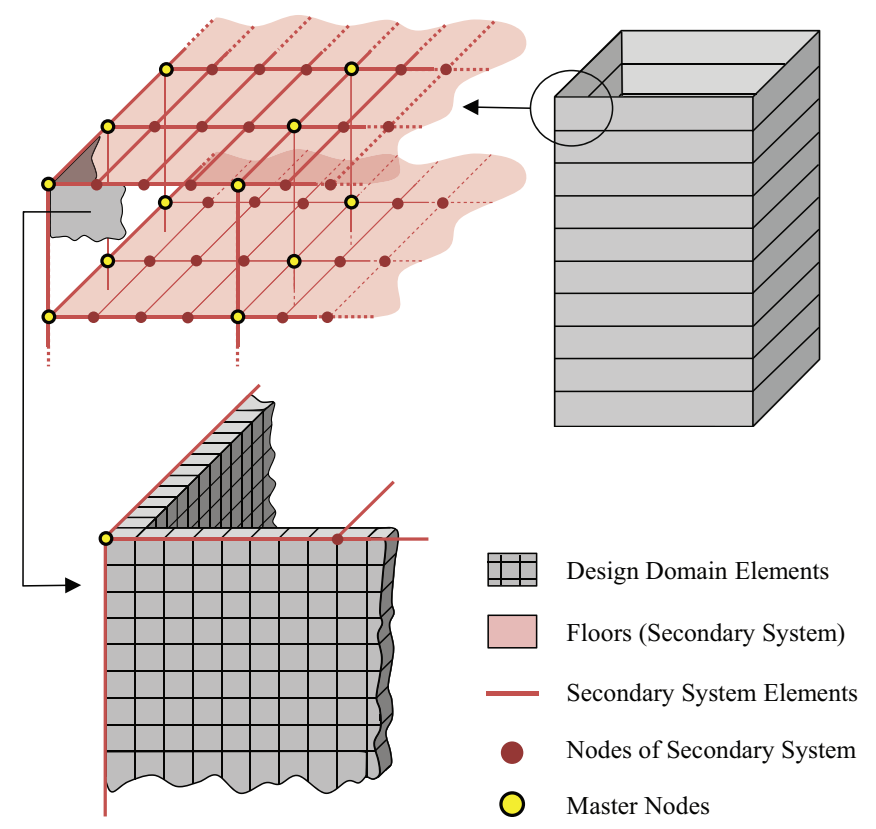

Figure 1: Schematic of the discretized design domain and complete FE model.

vector $\mathbf{y}$ through a local filter operator $\varphi_{e}$ (e.g. the H-filter [29]) such that $\boldsymbol{\rho}=\varphi_{e}(\mathbf{y}), n$ is the total number of elements composing the discretized design domain, $\Omega_{e}$ denotes the domain of element $e, V$ is the volume of material in the design domain, $G_{j}$ is the limit state function of interest for the $j$ th reliability constraint while $N$ is the total number of reliability constraints, $\mathrm{P}_{0_{j}}$ is the target failure probability for the $j$ th reliability constraint, $\mathbf{U}_{s}$ is a vector of uncertain structural parameters, $\mathbf{U}_{g}$ is a vector of uncertain parameters modeling the intensity and epistemic uncertainties of the stochastic excitation, and $\mathbf{X}$ is a white noise sequence specifying the stochastic excitation. In writing the problem setting of Eq. (1), realizations of the vectors $\mathbf{U}_{s}, \mathbf{U}_{g}$ and $\mathbf{X}$ are indicated in lowercase.

As mentioned in the introduction, the solution of the optimization defined in Eq. (1) is not trivial due to the considerable size of the design variable vector as well as the high-dimensional probabilistic integrals that define the reliability constraints. Indeed, the use of gradient-based algorithms is desirable for problems with large design variable vectors due to their efficiency; however, the implicit dependence of the reliability constraints on the design variables $\boldsymbol{\rho}$ significantly increases the computational effort needed to obtain 
their sensitivities. Although the use of approximate reliability methods (first and second order reliability methods) may decrease the computational effort needed to solve the reliability integrals in Eq. (1), the use of these techniques may be rendered impractical when the space of the vectors $\mathbf{U}_{s}, \mathbf{U}_{g}$ and $\mathbf{X}$ is high dimensional, e.g. systems subject to general stochastic excitation and constraints in terms of first excursion probabilities, due to difficulties in finding the "most probable point" [30]. This work is interested in defining an efficient solution strategy for these types of problems.

\section{Response analysis}

\subsection{Stochastic excitation}

In order to give a general description to the stochastic excitation $\ddot{z}_{g}$, the model proposed in [31] is used in this work. This model completely describes the excitation through a target amplitude spectrum $A\left(f ; \mathbf{u}_{g}\right)$, defining the frequency content of the excitation, and an envelope function, $e_{t}\left(t ; \mathbf{u}_{g}\right)$, that describes the potential non-stationarity in time of $\ddot{z}_{g}$. In particular, this model is well suited for modeling seismic ground motions. In this case, $A\left(f ; \mathbf{u}_{g}\right)$ and $e_{t}\left(t ; \mathbf{u}_{g}\right)$ are directly related to the intensity of the seismic event through the moment magnitude $M$ and the fault plane distance $r$ [31]. In general $A\left(f ; \mathbf{u}_{g}\right)$ will also depend on a number of parameters modeling the physics of the earthquake as well as the wave propagation process. These parameters are here grouped in the vector $\boldsymbol{\epsilon}$ and considered as random variables as suggested in [32]. Following this model, the vector $\mathbf{U}_{g}$ is given as $\left\{M, r, \boldsymbol{\epsilon}^{T}\right\}^{T}$.

In order to generate a discrete realization of $\ddot{z}_{g}$ of duration $T$ and time step $\Delta t$, the following steps may be followed:

1. Generate a white noise sequence $\mathbf{x}=\left[x(i \Delta t): i=1,2, \ldots, N_{T}\right]$ of total duration $T=N_{T} \Delta t$.

2. Multiply $\mathbf{x}$ by the envelope function $e_{t}\left(t ; \mathbf{u}_{g}\right)$ calibrated to a realization of $\mathbf{U}_{g}$.

3. Transform the resulting sequence into the frequency domain and normalize by the square root of the mean of the amplitude spectrum.

4. Multiply the normalized spectrum by the target amplitude spectrum $A\left(f ; \mathbf{u}_{g}\right)$.

5. Transform the normalized spectrum back into the time domain therefore generating a discrete realization of $\ddot{z}_{g}$ of total duration $T$ and time step $\Delta t$. 
More information on the specific models adopted in this work for modeling $A\left(f ; \mathbf{u}_{g}\right)$ as well as the components of $\boldsymbol{\epsilon}$ are reported in Appendix A.

\subsection{Response and damage models}

\subsubsection{Response model}

In order to describe the response of the system in a generic parameter $R$ (e.g. displacement, rotation, inter-story drift, stress) the following load-effect model is assumed:

$$
R\left(t ; \boldsymbol{\rho}, \mathbf{u}_{s}, \mathbf{u}_{g}, \mathbf{x}\right)=s \boldsymbol{\Gamma}_{R}^{T}(\boldsymbol{\rho}) \mathbf{K}(\boldsymbol{\rho}) \mathbf{z}\left(t ; \boldsymbol{\rho}, \mathbf{u}_{s}, \mathbf{u}_{g}, \mathbf{x}\right)
$$

where $s$ is an uncertain parameter modeling the epistemic uncertainty in the model of Eq. (2) while $\boldsymbol{\Gamma}_{R}$ is a vector of influence functions relating the dynamic response vector of the system, $\mathbf{z}(t)$, to the response parameter of interest $R$. In general, in order to estimate $\mathbf{z}(t)$, the full dynamic equilibrium equations need to be solved through direct integration. For linear systems, such as those of interest to this work, an alternative and generally more efficient approach is to use a classic modal framework. Under this assumption, $\mathbf{z}(t)$ can be estimated as (where any approximation is eliminated if all modes are considered):

$$
\mathbf{z}\left(t ; \boldsymbol{\rho}, \mathbf{u}_{s}, \mathbf{u}_{g}, \mathbf{x}\right) \approx \boldsymbol{\Phi}_{k}(\boldsymbol{\rho}) \mathbf{q}_{k}\left(t ; \boldsymbol{\rho}, \mathbf{u}_{s}, \mathbf{u}_{g}, \mathbf{x}\right)
$$

where $\boldsymbol{\Phi}_{k}=\left[\phi_{1}, \ldots, \phi_{k}\right]$ is a matrix containing the structure's first $k$ mode shape vectors while $\mathbf{q}_{k}=\left\{q_{1}(t), \ldots, q_{k}(t)\right\}^{T}$ is a vector containing the structure's first $k$ modal displacements at time $t$ determined from the solution of the following modal equation:

$$
\ddot{q}_{i}(t)+2 \xi_{i}\left(\mathbf{u}_{s}\right) \omega_{i}\left(\mathbf{u}_{s}\right) \dot{q}_{i}(t)+\omega_{i}^{2}\left(\mathbf{u}_{s}\right) q_{i}(t)=\frac{\boldsymbol{\phi}_{i}^{T} \mathbf{f}\left(t ; \mathbf{u}_{g}, \mathbf{x}\right)}{m_{i}}
$$

where $\xi_{i}, \omega_{i}$ and $m_{i}$ are the $i$ th mode's damping ratio, natural circular frequency and generalized mass respectively while $\mathbf{f}$ is the external loading vector. In particular, the structure's first $k$ mode shape vectors are calculated from the stiffness $\mathbf{K}$ and mass matrix $\mathbf{M}$ of the complete system through the resolution of the following standard eigenvalue problem:

$$
\left(\mathbf{K}-\omega_{i}^{2} \mathbf{M}\right) \phi_{i}
$$

while $\mathbf{f}$ is given by:

$$
\mathbf{f}\left(t ; \mathbf{u}_{g}, \mathbf{x}\right)=\mathbf{v}(\boldsymbol{\rho}) \ddot{z}_{g}\left(t ; \mathbf{u}_{g}, \mathbf{x}\right)=-\mathbf{M}(\boldsymbol{\rho}) \ddot{\boldsymbol{i}}_{g}\left(t ; \mathbf{u}_{g}, \mathbf{x}\right)
$$


where $\boldsymbol{i}$ is a vector of influence coefficients identifying the masses that participate in the directional excitation $\ddot{z}_{g}$. For efficiently solving the modal equations directly in the time domain, the procedure presented in [33] is adopted in this work.

It should be observed that in the proposed framework the uncertainties in the systems parameters, i.e. damping, stiffness and mass, are modeled directly at a modal level. While this is not as general as modeling the system uncertainties at the level of the damping, stiffness and mass matrices, it is an important step in this direction and has the advantage of being more easily calibrated. Indeed, there is in general a far better understanding of the uncertainties affecting the modal properties of a system than there is of those affecting the system matrices. This is especially true for damping $[34,35]$ which represents one of the most important parameters for dynamic systems. Having said this, explicit modeling of the uncertainties effecting the system matrices can be important $[15,16,19]$, and future development of this framework will focus on extending the proposed approach to a more general description of system uncertainty.

\subsubsection{Damage model}

For describing structural/non-structural damage to the system, it will be assumed that each component of interest (e.g. cladding component, interior partition, structural joint) is sensitive to a particular response parameter $R$ (e.g. inter-story drift, joint rotation, stress). The occurrence of a predefined damage state can then be described by the following damage ratio:

$$
D\left(\boldsymbol{\rho}, \mathbf{u}_{s}, \mathbf{u}_{g}, \mathbf{x}\right)=\max _{t \in[0, T]} \frac{\left|R\left(t ; \boldsymbol{\rho}, \mathbf{u}_{s}, \mathbf{u}_{g}, \mathbf{x}\right)\right|}{c}=\frac{\left|R\left(\hat{t} ; \boldsymbol{\rho}, \mathbf{u}_{s}, \mathbf{u}_{g}, \mathbf{x}\right)\right|}{c}
$$

where $\hat{t} \in[0, T]$ and is the instant in which $R$ assumes its largest absolute value while $c$ is the capacity of the component, i.e. the response level for which the predefined damage state occurs. In general, $c$ will be characterized by uncertainty, especially for non-structural components, and will here be

considered as a random variable belonging to the vector $\mathbf{U}_{s}$. By assuming the response model of Section 3.2.1, then $\mathbf{U}_{s}=\left\{C, S, \omega_{1}, \omega_{2}, \ldots, \omega_{k}, \xi_{1}, \xi_{2}, \ldots, \xi_{k}\right\}^{T}$.

\subsection{The reliability constraints}

The failure event of interest to the $j$ th reliability constraint can be stated as:

$$
F_{j}=\left\{G_{j}\left(\boldsymbol{\rho}, \mathbf{u}_{s}, \mathbf{u}_{g}, \mathbf{x}\right) \leq 0\right\}
$$


where $G_{j}$ is the limit state function given by:

$$
G_{j}\left(\boldsymbol{\rho}, \mathbf{u}_{s}, \mathbf{u}_{g}, \mathbf{x}\right)=\tilde{D}_{j}-D_{j}\left(\boldsymbol{\rho}, \mathbf{u}_{s}, \mathbf{u}_{g}, \mathbf{x}\right)
$$

where $\tilde{D}_{j}$ is the threshold value indicating the initiation of the damage state defined by $D_{j}$ and is generally taken equal to unity. Under these circumstances, the probability of failure is given by the following probabilistic integral:

$$
\begin{array}{r}
\mathrm{P}\left(F_{j}\right)=\mathrm{P}\left(G_{j}\left(\boldsymbol{\rho}, \mathbf{U}_{s}, \mathbf{U}_{g}, \mathbf{X}\right) \leq 0\right)= \\
\iint_{G_{j}\left(\boldsymbol{\rho}, \mathbf{u}_{s}, \mathbf{u}_{g}, \mathbf{x}\right) \leq 0} p\left(\mathbf{u}_{s}\right) p\left(\mathbf{u}_{g}\right) p(\mathbf{x}) \mathrm{d} \mathbf{u}_{s} \mathrm{~d} \mathbf{u}_{g} \mathrm{~d} \mathbf{x}
\end{array}
$$

where $p\left(\mathbf{u}_{s}\right), p\left(\mathbf{u}_{g}\right)$ and $p(\mathbf{x})$ are the joint probability density functions of the random vectors $\mathbf{U}_{s}, \mathbf{U}_{g}$ and $\mathbf{X}$ respectively. From how the damage measures of Eq. (7) are defined, it is evident that the probabilistic integrals of Eq. (10) represent the first excursion probability of the non-stationary damage measures $D_{j}$ across the thresholds $\tilde{D}_{j}$ during the event of duration $T$. For the problems of interest to this work, the integrals of Eq. (10) will be of high dimensions (in the order of thousands) primarily due to the vector $\mathbf{X}$ describing the stochastic excitation. For this reason, simulation methods are generally required for the evaluation of the integrals of Eq. (10) [36]. If Monte Carlo (MC) methods are used, then solutions can be estimated as:

$$
\mathrm{P}\left(G_{j}\left(\boldsymbol{\rho}, \mathbf{U}_{s}, \mathbf{U}_{g}, \mathbf{X}\right) \leq 0\right) \approx \frac{1}{N_{s}} \sum_{i=1}^{N_{s}} \mathcal{I}_{G_{j}}\left(\boldsymbol{\rho}, \mathbf{u}_{s}^{(i)}, \mathbf{u}_{g}^{(i)}, \mathbf{x}^{(i)}\right)
$$

where $N_{s}$ is the number of samples used in the simulation while $\mathcal{I}_{G_{j}}$ is an indicator function with $\mathcal{I}_{G_{j}}=1$ if the statement in Eq. (8) is true otherwise $\mathcal{I}_{G_{j}}=0$. The estimation of the integrals of Eq. (10), and therefore of the reliability constraints of the RBTO problem outlined in Eq. (1), requires the generation of $N_{s}$ samples of the damages measures of Eq. (7). These samples can be used to evaluate the following equivalent inverse constraints. Indeed, if it is assumed that the distribution functions, $F_{D_{j}}$, of $D_{j}$ are strictly monotonic (which holds true in nearly all cases of practical interest), then the following constraints are perfectly equivalent to the original ones (the probabilistic constraints of the problem outlined in Eq. (1)):

$$
D_{j}^{\left(\mathrm{P}_{0_{j}}\right)}(\boldsymbol{\rho})-\tilde{D}_{j} \leq 0
$$


where $D_{j}^{\left(\mathrm{P}_{0_{j}}\right)}$ is the damage measure threshold corresponding to the target failure probability $\mathrm{P}_{0_{j}}$. The evaluation of these constraints requires the evaluation of the thresholds $D_{j}^{\left(\mathrm{P}_{0_{j}}\right)}$ which can be achieved through the expression:

$$
D_{j}^{\left(\mathrm{P}_{0_{j}}\right)}(\boldsymbol{\rho})=F_{D_{j}}^{-1}\left(\mathrm{P}_{0_{j}} ; \boldsymbol{\rho}\right)
$$

where $F_{D_{j}}$ can be estimated directly from the MC samples of $D_{j}$.

The consideration of the inverse constraints of Eq. (12) does not afford any evident advantage over those of Eq. (1). Indeed, their evaluation still requires a full simulation to be carried out in order to estimate the thresholds $D_{j}^{\left(\mathrm{P}_{0_{j}}\right)}$. However, as will be shown in the following, a particularly convenient form can be given to the thresholds $D_{j}^{\left(\mathrm{P}_{0_{j}}\right)}$ and therefore to the constraints of Eq. (12).

\subsubsection{The reduced system}

Whether the original or inverse constraints are considered, their evaluation requires a simulation and therefore repeated evaluation of the complete FE model of the system within a dynamic setting. This can become especially cumbersome in topology optimization due to the sheer size of the FE models that must be considered in order to give an adequate resolution to the design domain. This problem can be alleviated by taking advantage of how the mass of the complete system is lumped at a reduced number of degrees of freedom (Section 2.1). Indeed, by reducing the stiffness matrix, $\mathbf{K}$, to these degrees of freedom through static condensation while eliminating the lines and columns of the mass matrix, $\mathbf{M}$, corresponding to degrees of freedom with zero mass (therefore unloaded), the following modal equations can be defined in terms of the reduced stiffness and mass matrices:

$$
\ddot{q}_{i}(t)+2 \xi_{i}\left(\mathbf{u}_{s}\right) \tilde{\omega}_{i}\left(\mathbf{u}_{s}\right) \dot{q}_{i}(t)+\tilde{\omega}_{i}^{2}\left(\mathbf{u}_{s}\right) q_{i}(t)=\frac{\tilde{\boldsymbol{\phi}}_{i}^{T} \tilde{\mathbf{f}}\left(t ; \mathbf{u}_{g}, \mathbf{x}\right)}{\tilde{m}_{i}}
$$

where $\tilde{\omega}_{i}, \tilde{m}_{i}$ and $\tilde{\phi}_{i}$ are the $i$ th modes natural circular frequency, generalized mass and mode shape estimated from the reduced stiffness, $\tilde{\mathbf{K}}$, and mass, $\tilde{\mathbf{M}}$, matrices respectively while $\tilde{\mathbf{f}}$ is the reduced excitation vector obtained from $\mathbf{f}$ (Eq. (6)) by simply eliminating the lines corresponding to degrees of freedom with zero excitation. 
If the modal damping ratios are taken equal between the reduced and complete systems, the following equality holds:

$$
\begin{aligned}
R\left(t ; \boldsymbol{\rho}, \mathbf{u}_{s}, \mathbf{u}_{g}, \mathbf{x}\right) & =s \boldsymbol{\Gamma}_{R}^{T}(\boldsymbol{\rho}) \mathbf{K}(\boldsymbol{\rho}) \boldsymbol{\Phi}_{k}(\boldsymbol{\rho}) \mathbf{q}_{k}\left(t ; \boldsymbol{\rho}, \mathbf{u}_{s}, \mathbf{u}_{g}, \mathbf{x}\right) \\
& =s \tilde{\boldsymbol{\Gamma}}_{R}^{T}(\boldsymbol{\rho}) \tilde{\mathbf{K}}(\boldsymbol{\rho}) \tilde{\boldsymbol{\Phi}}_{k}(\boldsymbol{\rho}) \tilde{\mathbf{q}}_{k}\left(t ; \boldsymbol{\rho}, \mathbf{u}_{s}, \mathbf{u}_{g}, \mathbf{x}\right)
\end{aligned}
$$

where $\tilde{\boldsymbol{\Gamma}}_{R}$ is the influence function of $R$ in the reduced system obtained by eliminating the components of $\boldsymbol{\Gamma}_{R}$ corresponding to the unloaded degrees of freedom, $\tilde{\boldsymbol{\Phi}}_{k}$ is the mode shape matrix of order $k$ estimated in the reduced system while $\tilde{\mathbf{q}}_{k}$ is the vector of modal displacements estimated from Eq. (14). Equation (15) is an exact relationship and illustrates how the use of the reduced system does not introduce any approximations. The advantage of considering the reduced system in place of the complete system (secondary system plus design domain) is that it reduces the size of the FE model that needs to be used during each simulation by several orders of magnitude.

\section{The proposed RBTO algorithm}

This section will focus on developing an efficient approach for the solution of the RBTO problem outlined in Section 2. In particular, the approach is based on augmenting the simulation process while adopting the load-effect model of Section 3.2.1. This allows for the definition of an equivalent form for the inverse constraints of Section 3.3 that may be used to define an optimization sub-problem. In defining this equivalent form for the constraints of Eq. (12), the basic idea that will be explored is the possibility of writing the damage measure thresholds $D_{j}^{\left(\mathrm{P}_{0_{j}}\right)}$ in terms of equivalent static load profiles (one for each constraint) that is independent of small changes in the material density design variable vector $\boldsymbol{\rho}$. This will allow the probabilistic and dynamic optimization problem of Eqs. (1) to be replaced by a sequence of classic deterministic and static optimization problems. The fundamental step in ensuring the success of this idea is the definition of the equivalent static load profiles that must retain physical meaning. In this respect, the model that will be derived in this work will be based on state-of-the-art models developed for describing equivalent static loads for wind excited dynamic systems $[37,38]$. In the following, the notation $\boldsymbol{\rho}_{0}$ will be used to indicate a fixed value of the design variable vector. 


\subsection{The equivalent constraints}

In order to derive the equivalent constraints, it is first necessary to consider the following variable that can be defined for each realization of the vectors $\mathbf{U}_{s}, \mathbf{U}_{g}$ and $\mathbf{X}$ and therefore for each simulation point:

$$
\begin{aligned}
\tilde{\boldsymbol{\Upsilon}}_{j}\left(\boldsymbol{\rho}_{0}, \mathbf{u}_{s}, \mathbf{u}_{g}, \mathbf{x}\right)=\overline{\tilde{\mathcal{F}}}_{j}^{(T)}\left(\boldsymbol{\rho}_{0}, \mathbf{u}_{s}, \mathbf{u}_{g}, \mathbf{x}\right) \\
+\gamma_{R_{j}}\left(\boldsymbol{\rho}_{0}, \mathbf{u}_{s}, \mathbf{u}_{g}, \mathbf{x}\right) \frac{\mathbf{C}_{\tilde{\mathcal{F}}_{j}}^{(T)}\left(\boldsymbol{\rho}_{0}, \mathbf{u}_{s}, \mathbf{u}_{g}, \mathbf{x}\right) \tilde{\boldsymbol{\Gamma}}_{R_{j}}\left(\boldsymbol{\rho}_{0}\right)}{\sigma_{R_{j}}^{(T)}\left(\boldsymbol{\rho}_{0}, \mathbf{u}_{s}, \mathbf{u}_{g}, \mathbf{x}\right)}
\end{aligned}
$$

where $\sigma_{R_{j}}^{(T)}$ is the standard deviation, in the sense of the time statistics carried out in a time window of duration $T$, of the response parameter $R$; $\gamma_{R_{j}}$ is the number of standard deviations that the largest absolute value of the response process $R\left(t ; \boldsymbol{\rho}_{0}, \mathbf{u}_{s}, \mathbf{u}_{g}, \mathbf{x}\right)$ (for $t \in[0, T]$ ) is from its mean value; while $\overline{\mathcal{F}}_{j}^{(T)}$ and $\mathbf{C}_{\tilde{\mathcal{F}}_{j}}^{(T)}$ are the mean and covariance matrix (again in the sense of the time statistics carried out in a time window of duration $T$ ) of the following vector valued stochastic process:

$$
\tilde{\mathcal{F}}\left(t ; \boldsymbol{\rho}_{0}, \mathbf{u}_{s}, \mathbf{u}_{g}, \mathbf{x}\right)=s \tilde{\mathbf{K}}\left(\boldsymbol{\rho}_{0}\right) \tilde{\boldsymbol{\Phi}}_{k}\left(\boldsymbol{\rho}_{0}\right) \tilde{\mathbf{q}}_{k}\left(t ; \boldsymbol{\rho}_{0}, \mathbf{u}_{s}, \mathbf{u}_{g}, \mathbf{x}\right)
$$

The physical meaning of Eq. (17) can be found in its close relationship to the most probably load distribution (including inertial effects) to generate the largest absolute value of the response process $R(t)$ for $t \in[0, T]$. Indeed, Eq. (17) can be seen as a time varying loading vector that if applied to the system of stiffness $\tilde{\mathbf{K}}$ will generate the exact dynamic response estimated from the modal analysis. From [37, 39], it follows that Eq. (16) is therefore related the most probable instantaneous load distribution to generate the absolute largest value of $R$ in $[0, T]$. In particular, from how $\tilde{\Upsilon}_{j}$ is defined the following must hold (as demonstrated in $[37,38]$ ):

$$
\left|R\left(\hat{t} ; \boldsymbol{\rho}_{0}, \mathbf{u}_{s}, \mathbf{u}_{g}, \mathbf{x}\right)\right|=\tilde{\boldsymbol{\Gamma}}_{R_{j}}^{T}\left(\boldsymbol{\rho}_{0}\right) \tilde{\boldsymbol{\Upsilon}}_{j}\left(\boldsymbol{\rho}_{0}, \mathbf{u}_{s}, \mathbf{u}_{g}, \mathbf{x}\right)
$$

Alternatively, and maybe more intuitively, $\tilde{\Upsilon}_{j}$ can be defined as:

$$
\tilde{\boldsymbol{\Upsilon}}_{j}\left(\boldsymbol{\rho}_{0}, \mathbf{u}_{s}, \mathbf{u}_{g}, \mathbf{x}\right)=( \pm 1) \tilde{\mathcal{F}}_{j}\left(\hat{t} ; \boldsymbol{\rho}_{0}, \mathbf{u}_{s}, \mathbf{u}_{g}, \mathbf{x}\right)
$$

and therefore as the sign corrected value of the vector $\tilde{\mathcal{F}}_{j}$ that results in the largest absolute value of $R$ in $[0, T]$. The philosophy behind defining $\tilde{\Upsilon}_{j}$ in 
terms of the time statistics of $R_{j}$, and therefore in the form of Eq. (16), is to relate the largest absolute response $R_{j}(\hat{t})$ to the response process occurring in the window of duration $T$ instead of it depending exclusively on the response of the system in $\hat{t}$, as is the case for the formulation of Eq. (19). It is believed that this will reduce the sensitivity of $R_{j}(\hat{t})$ to small changes in the dynamic properties of the system. The relative performance of the two methods will be investigated in the first case study of this work where the formulation of Eq. (16) will be termed "Method 1" and that of Eq. (19) will be termed "Method 2".

By writing the random capacity, $C_{j}$, as $\bar{C}_{j}\left(1+\delta_{C_{j}} C_{n, j}\right)$ with $\bar{C}_{j}$ the mean/nominal value of $C_{j}, \delta_{C_{j}}$ the coefficient of variation of $C_{j}$ and $C_{n, j}$ the standardized (zero mean and unit standard deviation) capacity, the following vector can be defined:

$$
\tilde{\boldsymbol{\psi}}_{j}\left(\boldsymbol{\rho}_{0}, \mathbf{u}_{s}, \mathbf{u}_{g}, \mathbf{x}\right)=\frac{\tilde{\boldsymbol{\Upsilon}}_{j}\left(\boldsymbol{\rho}_{0}, \mathbf{u}_{s}, \mathbf{u}_{g}, \mathbf{x}\right)}{1+\delta_{C_{j}} c_{n, j}}
$$

where $c_{n, j}$ is the realization of $C_{n, j}$ contained in $\mathbf{u}_{s}$ while $\tilde{\boldsymbol{\Upsilon}}_{j}$ can be calculated using either "Method 1" or "Method 2". The interest in defining $\tilde{\boldsymbol{\psi}}_{j}$ is that the following holds for the damage measure $D_{j}$ associated with the sample point defined by the realizations $\mathbf{u}_{s}, \mathbf{u}_{g}$ and $\mathbf{x}$ :

$$
\begin{aligned}
D_{j}\left(\boldsymbol{\rho}_{0}, \mathbf{u}_{s}, \mathbf{u}_{g}, \mathbf{x}\right) & =\frac{\left|R\left(\hat{t} ; \boldsymbol{\rho}_{0}, \mathbf{u}_{s}, \mathbf{u}_{g}, \mathbf{x}\right)\right|}{\bar{C}_{j}\left(1+\delta_{C_{j}} c_{n, j}\right)} \\
& =\frac{\tilde{\boldsymbol{\Gamma}}_{R_{j}}^{T}\left(\boldsymbol{\rho}_{0}\right) \tilde{\boldsymbol{\psi}}_{j}\left(\boldsymbol{\rho}_{0}, \mathbf{u}_{s}, \mathbf{u}_{g}, \mathbf{x}\right)}{\bar{C}_{j}}
\end{aligned}
$$

In particular, by now considering that a MC simulation with $N_{s}$ samples is carried out in $\boldsymbol{\rho}_{0}$ with the aim of evaluating the constraints of Eq. (12), then as a by-product of this process the following quantities can be defined:

$$
\begin{gathered}
\overline{\tilde{\boldsymbol{\psi}}}_{j}\left(\boldsymbol{\rho}_{0}\right)=\frac{1}{N_{s}} \sum_{i=1}^{N_{s}} \tilde{\boldsymbol{\psi}}_{j}\left(\boldsymbol{\rho}_{0}, \mathbf{u}_{s}^{(i)}, \mathbf{u}_{g}^{(i)}, \mathbf{x}^{(i)}\right) \\
\mathbf{C}_{\tilde{\boldsymbol{\psi}}_{j}}\left(\boldsymbol{\rho}_{0}\right)=\frac{1}{N_{s}-1} \sum_{i=1}^{N_{s}}\left(\tilde{\boldsymbol{\psi}}_{j}\left(\boldsymbol{\rho}_{0}, \mathbf{u}_{s}^{(i)}, \mathbf{u}_{g}^{(i)}, \mathbf{x}^{(i)}\right)-\overline{\tilde{\boldsymbol{\psi}}}_{j}\left(\boldsymbol{\rho}_{0}\right)\right) \\
\times\left(\tilde{\boldsymbol{\psi}}_{j}\left(\boldsymbol{\rho}_{0}, \mathbf{u}_{s}^{(i)}, \mathbf{u}_{g}^{(i)}, \mathbf{x}^{(i)}\right)-\overline{\tilde{\boldsymbol{\psi}}}_{j}\left(\boldsymbol{\rho}_{0}\right)\right)^{T}
\end{gathered}
$$


where $\overline{\tilde{\boldsymbol{\psi}}}_{j}$ is the sample mean of $\tilde{\boldsymbol{\psi}}_{j}$ while $\mathbf{C}_{\tilde{\boldsymbol{\psi}}_{j}}$ is the sample covariance of $\tilde{\boldsymbol{\psi}}_{j}$. These quantities allow the following vector to be defined:

$$
\tilde{\boldsymbol{\Psi}}_{j}\left(\mathrm{P}_{0_{j}}, \boldsymbol{\rho}_{0}\right)=\overline{\boldsymbol{\psi}}_{j}\left(\boldsymbol{\rho}_{0}\right)+\gamma_{D_{j}}\left(\mathrm{P}_{0_{j}}, \boldsymbol{\rho}_{0}\right) \frac{\mathbf{C}_{\tilde{\boldsymbol{\psi}}_{j}}\left(\boldsymbol{\rho}_{0}\right) \tilde{\boldsymbol{\Gamma}}_{R_{j}}\left(\boldsymbol{\rho}_{0}\right)}{\sigma_{D_{j}}\left(\boldsymbol{\rho}_{0}\right) \bar{C}_{j}}
$$

where $\sigma_{D_{j}}$ is the sample standard deviation of $D_{j}$ while $\gamma_{D_{j}}$ is the number of standard deviations that the threshold $D_{j}^{\left(\mathrm{P}_{0_{j}}\right)}$ is from the sample mean, $\mu_{D_{j}}$, of $D_{j}$ and can be estimated simply as:

$$
\gamma_{D_{j}}\left(\boldsymbol{\rho}_{0}\right)=\frac{D_{j}^{\left(\mathrm{P}_{0_{j}}\right)}-\mu_{D_{j}}\left(\boldsymbol{\rho}_{0}\right)}{\sigma_{D_{j}}\left(\boldsymbol{\rho}_{0}\right)}
$$

where $D_{j}^{\left(\mathrm{P}_{0_{j}}\right)}$ is estimated as indicated in Section 3.3. The physical meaning of Eq. (24) can be understood by recalling that for each event the variable $\tilde{\Upsilon}_{j}$ (Eq. (16)) is related to the most probable load distribution causing the peak seismic demand. By observing the similarity in structure between Eq. (16)-single event-and Eq. (24)-all events-, $\tilde{\Psi}_{j}$ will be related to the most probable load distribution that generates a demand level of $D_{j}^{\left(\mathrm{P}_{0_{j}}\right)}$ over all the seismic events. In particular, because for each sample of $\tilde{\boldsymbol{\psi}}_{j}$ Eq. (21) holds, then the following must hold (see Appendix B):

$$
D_{j}^{\left(\mathrm{P}_{0_{j}}\right)}\left(\boldsymbol{\rho}_{0}\right)=\frac{\tilde{\boldsymbol{\Gamma}}_{R_{j}}^{T}\left(\boldsymbol{\rho}_{0}\right) \tilde{\boldsymbol{\Psi}}_{j}\left(\mathrm{P}_{0_{j}}, \boldsymbol{\rho}_{0}\right)}{\bar{C}_{j}}
$$

which illustrates how the thresholds $D_{j}^{\left(\mathrm{P}_{0_{j}}\right)}$ can be written in terms of the vector $\tilde{\boldsymbol{\Psi}}_{j}$ which plays the part of a static load distribution. The apparent simplicity of the relationship derived above, which will be taken advantage of in the next section, should not overshadow the complexity of $\tilde{\mathbf{\Psi}}_{j}$ which not only incorporates the probabilistic characteristics of the excitation and system parameters, but also the dynamic nature of the system.

As the above notation suggests, the simulation is carried out in the reduced system. In order to transfer $\tilde{\mathbf{\Psi}}_{j}$ to the complete system, it is simply necessary to add zeros to $\tilde{\boldsymbol{\Psi}}_{j}$ in correspondence to the degrees of freedom of the complete system that have zero mass:

$$
\Psi_{j}^{i}\left(\mathrm{P}_{0_{j}}, \boldsymbol{\rho}_{0}\right)= \begin{cases}\tilde{\Psi}_{j}^{k}\left(\mathrm{P}_{0_{j}}, \boldsymbol{\rho}_{0}\right), & i=k \in \Xi \\ 0, & i \neq k \in \Xi\end{cases}
$$


where $\Xi$ is the set of degrees of freedom defining the master nodes while $i=1, \ldots, D_{\text {dof }}$ with $D_{\text {dof }}$ indicating the total number of degrees of freedom of the complete system. It is now possible to write the searched after equivalent form of the first excursion reliability constraints:

$$
\frac{\boldsymbol{\Gamma}_{R_{j}}^{T}\left(\boldsymbol{\rho}_{0}\right) \boldsymbol{\Psi}_{j}\left(\mathrm{P}_{0_{j}}, \boldsymbol{\rho}_{0}\right)}{\bar{C}_{j}}-\tilde{D}_{j} \leq 0
$$

which may also be written, as is typical of topology optimization problems, in terms of the displacement vector as:

$$
\frac{\boldsymbol{\Lambda}_{j}^{T}\left(\boldsymbol{\rho}_{0}\right) \mathbf{z}_{j}\left(\boldsymbol{\rho}_{0}\right)}{\bar{C}_{j}}-\tilde{D}_{j} \leq 0
$$

where the displacement vector $\mathbf{z}_{j}$ is obtained from the static equilibrium equation $\mathbf{K}\left(\boldsymbol{\rho}_{0}\right) \mathbf{z}\left(\boldsymbol{\rho}_{0}\right)=\boldsymbol{\Psi}_{j}\left(\mathrm{P}_{0_{j}}, \boldsymbol{\rho}_{0}\right), \boldsymbol{\Lambda}_{j}$ is a vector of constants extracting the response $R$ and is given as $\boldsymbol{\Lambda}_{j}\left(\boldsymbol{\rho}_{0}\right)=\mathbf{K}\left(\boldsymbol{\rho}_{0}\right) \boldsymbol{\Gamma}_{R_{j}}\left(\boldsymbol{\rho}_{0}\right)$. In particular, if $R$ is a displacement/rotation, or a relative displacement/rotation, then $\boldsymbol{\Lambda}_{j}$ becomes independent of $\boldsymbol{\rho}_{0}$. The fundamental property of the formulation of Eqs. (28) or (29) that will be explored in the following is how the probabilistic and dynamic nature of the system and excitation are now entirely contained in $\boldsymbol{\Psi}_{j}$ which can be estimated as a simple by-product of the simulation process carried out in $\boldsymbol{\rho}_{0}$. Indeed, all other quantities appearing in Eqs. (28) or (29) are in terms of their mean/nominal quantities.

\subsection{The optimization sub-problem}

As the notation of the previous section indicates, $\boldsymbol{\Psi}_{j}$ depends on $\boldsymbol{\rho}$ and therefore on where in the design space it is calculated. It is also an implicit function of $\boldsymbol{\rho}$. Therefore, the direct use of the equivalent constraints of Eqs. (28) or (29) does not simplify the optimization problem of Eq. (1). However, it can be observed that $\boldsymbol{\Psi}_{j}$ strongly depends on the external stochastic excitation, herein denoted as $\ddot{z}_{g}$, which is independent of $\boldsymbol{\rho}$. Indeed $\boldsymbol{\Psi}_{j}$ depends

largely on $\tilde{\Upsilon}_{j}$ (Eq. (16)), which in turn depends significantly on $\tilde{\mathcal{F}}$ (Eq. (17)). By observing that $\tilde{\mathcal{F}}$ can be written as:

$$
\begin{aligned}
\tilde{\mathcal{F}}\left(t ; \boldsymbol{\rho}_{0}, \mathbf{u}_{s}, \mathbf{u}_{g}, \mathbf{x}\right)= & s \tilde{\mathbf{M}}\left(\boldsymbol{\rho}_{0}\right) \boldsymbol{i} \ddot{z}_{g}\left(t ; \mathbf{u}_{g}, \mathbf{x}\right) \\
& +s \tilde{\mathbf{K}}\left(\boldsymbol{\rho}_{0}\right) \tilde{\boldsymbol{\Phi}}_{k}\left(\boldsymbol{\rho}_{0}\right) \tilde{\mathbf{q}}_{k, r}\left(t ; \boldsymbol{\rho}_{0}, \mathbf{u}_{s}, \mathbf{u}_{g}, \mathbf{x}\right)
\end{aligned}
$$


where $\tilde{\mathbf{q}}_{k, r}$ is the vector of resonant modal responses with components given by:

$$
\tilde{q}_{i, r}(t)=\tilde{q}_{i}(t)-\frac{\tilde{\boldsymbol{\phi}}_{i}^{T} \mathbf{f}(t)}{\tilde{m}_{i} \tilde{\omega}_{i}^{2}}
$$

it is evident that $\tilde{\mathcal{F}}$ has a resonant and background quasi-static component (first term of the right hand side of Eq. (30)). If it is now observed that the majority of mass in a typical building is carried, then the term $\tilde{\mathbf{M}}\left(\boldsymbol{\rho}_{0}\right)$ will be relatively insensitive to changes in $\boldsymbol{\rho}$. Therefore the background component of $\tilde{\mathcal{F}}$ can be considered independent of $\boldsymbol{\rho}_{0}$ which ultimately results in a weakening of the dependency of $\boldsymbol{\Psi}_{j}$ for small changes in $\boldsymbol{\rho}$ around $\boldsymbol{\rho}_{0}$. It would therefore seem reasonable to consider $\boldsymbol{\Psi}_{j}$ relatively insensitive to small changes around $\boldsymbol{\rho}_{0}$. This allows the optimization problem of Eq. (1) to be written as:

$$
\begin{array}{ll}
\min _{\boldsymbol{\rho}} & V(\boldsymbol{\rho})=\sum_{e=1}^{n} \int_{\Omega_{e}} \rho_{e} \mathrm{~d} \Omega \\
\text { s.t. } & \frac{\boldsymbol{\Lambda}_{j}^{T}(\boldsymbol{\rho}) \mathbf{z}(\boldsymbol{\rho})}{\bar{C}_{j}}-\tilde{D}_{j} \leq 0, \quad j=1, \ldots, N \\
& 0 \leq \rho_{e} \leq 1
\end{array}
$$

where $\mathbf{z}$ are obtained from the static equilibrium equation $\mathbf{K}(\boldsymbol{\rho}) \mathbf{z}(\boldsymbol{\rho})=$ $\boldsymbol{\Psi}_{j}\left(\mathrm{P}_{0_{j}}, \boldsymbol{\rho}_{0}\right)$. Because all quantities that appear in the above formulation take their mean/nominal value while $\boldsymbol{\Psi}_{j}$ is now treated as a constant, the above optimization problem can be completely solved without invoking any probabilistic analysis. Therefore the optimization loop has been effectively decoupled from the probabilistic analysis. In particular, looking at the mathematical structure of the optimization problem of Eq. (32), it is evident that it represents a classic topology optimization problem with $\boldsymbol{\Psi}_{j}$ acting as a static load profile. Therefore, any of the well developed optimization algorithms can be used to find solutions.

The constraints are exact only in $\boldsymbol{\rho}_{0}$, i.e. where $\boldsymbol{\Psi}_{j}$ is estimated. Therefore, the formulation of Eq. (32) only approximately decouples the probabilistic analysis from the optimization loop. This implies that the overall optimization process will consist in formulating and solving a sequence of optimization problems of the type shown in Eq. (32) which can therefore be considered an optimization sub-problem. Commencing in an arbitrary point, 
$\boldsymbol{\rho}_{0}$, of the the design space, the overall optimization process can be described as follows:

1. Carry out a simulation in $\boldsymbol{\rho}_{0}$ and formulate the sub-problem of Eq. (32).

2. Solve the sub-problem of step 1 using any topology optimization algorithm developed for static and deterministic problems (e.g. Method of Moving Asymptotes [40]). This step will yield a possible optimum point $\boldsymbol{\rho}_{1}$.

3. Check for overall convergence defined as $\boldsymbol{\rho}_{1}=\boldsymbol{\rho}_{0}$. If this is true, terminate otherwise move to step 4.

4. Update $\boldsymbol{\rho}_{0}$ by setting it equal to $\boldsymbol{\rho}_{1}$ and return to step 1.

In the following, each formulation and resolution of a sub-problem will be termed a design cycle.

The key properties of the proposed sequential optimization algorithm are: 1) each formulation of the sub-problem requires only one probabilistic analysis to be carried out with the aim of simply evaluating the reliability constraints (i.e. no additional sensitivity analysis is needed nor any global or local exploration of the design space); 2) the sub-problem takes on a classic static and deterministic form and therefore can be extremely efficiently solved: 3) the equivalent constraints of the sub-problem are exact in the point in which the sub-problem is formulated therefore ensuring that the final converged solution rigorously meets the original first excursion reliability constraints.

\section{Applications}

Two case studies are presented in this section with the aim of illustrating the applicability of the proposed RBTO algorithm to structures subject to non-stationary stochastic earthquake excitation. The case studies are focused on the topology design of the lateral load-resisting system of multistory planar frames. In particular, the first case study considers a small structure for the purpose of initial investigation while the second case study focus on a more practical design example represented by a mid-rise building.

\subsection{Excitation}

The stochastic excitation was simulated using the model of Section 3.1. In particular the amplitude spectrum $A\left(f ; \mathbf{U}_{g}\right)$ and envelope function $e_{t}\left(t ; \mathbf{U}_{g}\right)$ 

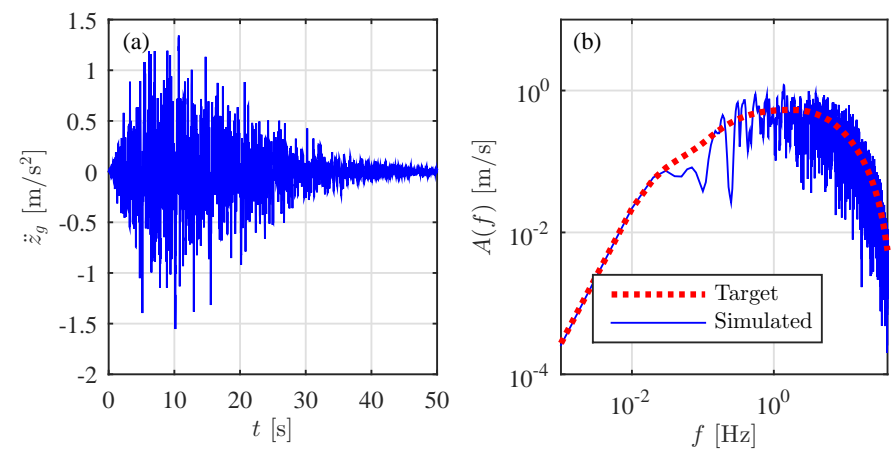

Figure 2: Illustrative sample of the non-stationary excitation: (a) time history of the first 50 s; (b) Amplitude spectrum.

were calibrated following the models described in Appendix A. This results in $\boldsymbol{\epsilon}=\left\{f_{a}, f_{b}, e, h, \kappa_{0}, f_{\max }, \lambda_{t}, \eta_{t}, T_{w}\right\}^{T}$ where the definition of each component can be found in Appendix A. The distributions considered for each component are those suggested in Vetter and Taflanidis [41] and reported for completeness in Table 1 . The other parameters of the model took the values: $R_{\Phi}=0.55 ; \rho_{s}=2.8 \mathrm{~g} / \mathrm{cm}^{3} ; c_{Q}=\beta_{s}=3.5 \mathrm{~km} / \mathrm{s} ; Q(f)=180 f^{0.45}$. The geometric spreading function $Z(R)$ was defined as:

$$
Z(R)= \begin{cases}1 / R & R \leq 70 \mathrm{~km} \\ 1 / 70 & R>70 \mathrm{~km}\end{cases}
$$

The intensity of the events were defined by the fault plane distance $r$, which was taken as a log-normal random variable with mean $30 \mathrm{~km}$ and coefficient of variation 0.4 , and the moment magnitude $M$, which was taken as the following truncated exponential distribution with $M_{\min }=5$ and $M_{\max }=8$ [42]:

$$
p(M)=\frac{\beta \exp \left[-\beta\left(M-M_{\min }\right)\right]}{1-\exp \left[-\beta\left(M_{\max }-M_{\min }\right)\right]}
$$

where the regional seismicity factor was taken as $\beta=1.0 \ln [10]$. The duration of the simulated stochastic ground accelerations was $T=100 \mathrm{~s}$ with $\Delta t=$ $0.005 \mathrm{~s}$. The total number of components, $N_{T}$, of the white noise sequences, $\mathbf{X}$, was therefore 20001. Figure 2 shows a typical realization of the nonstationary excitation. 
Table 1: Suggested distributions for $\boldsymbol{\epsilon}$.

\begin{tabular}{llll}
\hline Variable & P1* & P2 $^{* *}$ & Distribution \\
\hline$f_{a}$ & Eq. (A.6) & 0.25 & Log-normal \\
$f_{b}$ & Eq. (A.7) & 0.25 & Log-normal \\
$e$ & Eq. (A.8) & 0.25 & Log-normal \\
$h$ & Eq. (A.10) & 0.25 & Log-normal \\
$\kappa_{0}$ & 0.02 & 0.04 & Uniform \\
$f_{\text {max }}$ & $25 \mathrm{~Hz}$ & 0.30 & Log-normal \\
$\lambda_{t}$ & 0.2 & 0.40 & Log-normal \\
$\eta_{t}$ & 0.05 & 0.40 & Log-normal \\
$T_{w}$ & Eq. (A.19) & 0.40 & Log-normal \\
\hline${ }^{*}$ Median for Normal, Log-normal distributions; \\
Lower bound for Uniform distribution \\
** C.o.V. for Normal, Log-normal distributions; \\
Upper bound for Uniform distribution \\
\hline \multicolumn{4}{l}{}
\end{tabular}

\subsection{Performance objectives}

For each of the following case studies, performance was measured through damage ratios (as defined in Eq. (7)) set in terms of the drift response occurring at each floor of the frame. The associated capacity, $C$, was taken as a lognormal random variable with mean/nominal value, $\bar{C}$, equal to $1 / 400$ of the floor-to-floor height and coefficient of variation $\delta_{C}=0.01$. In calculating the response of the structures through the load-effect model of Section 3.2.1, the damping ratios, $\xi_{i}$, were taken as independent log-normal random variables with mean/nominal value $1.0 \%$ and coefficient of variation 0.3 . The natural frequencies $\omega_{i}$ were also considered as independent log-normal random variables with however the mean/nominal value derived from the mean/nominal stiffness and mass matrices of the complete system while the coefficient of variation was set equal to 0.01 . The uncertain parameter $S$ was taken as a normally distributed random variable with unit mean and coefficient of variation 0.025 .

All optimization problems commenced from an initial design defined by all the components of $\boldsymbol{\rho}$ taking the value of 0.5. For solving the sub-problems, the Solid Isotropic Material with Penalization (SIMP) method was used with a penalty of $5[43,44]$ while the Method of Moving Asymptotes [40] was used as the optimization algorithm. 


\subsection{Case Study 1}

This case study is focused on the topology optimization of the three story planar frame shown in Fig. 3(a). In particular, the aim is to investigate the convergence properties of the proposed optimization algorithm on a simple problem where the optimum solution can also be guessed through engineering intuition. The difference between adopting "Method 1" or "Method 2" during the definition of $\tilde{\Upsilon}_{j}$ (see Section 4.1) was also investigated. Reliability constraints are considered in terms of the drift responses occurring at the first, second and third floors. For each of the three constraints the critical threshold was taken as $\tilde{D}=1$. The initial structure for this case study is shown in Fig. 3(a). In particular, $w=5 \mathrm{~m}, h=15 \mathrm{~m}$, and $h_{\text {floor }}=5$ m. The design domain, indicated by the shaded gray area in Fig. 3(a), is discretized using 50 planar bilinear quadrilateral (Q4) elements in the horizontal direction and 150 planar Q4 elements in the vertical direction $(0.1 \mathrm{~m} \mathrm{x}$ $0.1 \mathrm{~m}$ with a thickness of $0.1 \mathrm{~m}$ ) for a total of 7500 elements. Symmetry was imposed about the vertical centerline of the structure therefore leading to a total of 3750 design variables. The planar elements were given the material properties of reinforced concrete with a Young's modulus of 21 GPa (in place of reinforced concrete any other material could be considered). A linear density filter [29] with a radius $r_{\min }=0.3 \mathrm{~m}$ was used during the optimization. The floor beams and columns of the secondary system were taken as W8×10 members with the properties of steel (Young's modulus $199.9 \mathrm{GPa}$ ). Their locations are indicated by the horizontal and vertical lines in Fig. 3(a), respectively. The secondary members were modeled using beam elements each with the length of a planar element $(0.1 \mathrm{~m})$ therefore leading to a total of 150 beam elements on each column line and 50 beam elements at each floor. In order to combine the two element types (Q4 and beam elements), the approach indicated in [5] was adopted. In this approach, the beam elements are attached at every node of the quadrilateral mesh therefore forcing the quadrilateral nodes to translate together with the beam elements (i.e. the translations of the beam elements are transferred to the Q4 elements). Two master nodes were considered at each floor location as shown in Fig. 3(a) therefore resulting in a total of six degrees of freedom for the reduced system. No additional carried mass was considered while the first three vibration modes were used in the response analysis resulting in a participating mass of over $99.99 \%$ in all the following examples. 


\subsubsection{Case Study 1: baseline structure}

The baseline system for this case study was defined considering the uncertainties reported in Sections 5.1 and 5.2. In particular, 75000 samples were considered in the Monte Carlo simulations carried out at each design cycle while the target first excursion probabilities in $[0, T]$ (the duration of the event) were taken as $\mathrm{P}_{0_{j}}=0.001$. Figures $3(\mathrm{~b})$ and (c) show the final topologies obtained using the proposed optimization scheme calibrated considering Method 1 or Method 2. As can be seen, the optimal topology is an X-bracing system, a solution also observed by Chun et al. [23] for a design domain with the same dimensions. This solution is in line with engineering intuition and is a first indication of the overall validity of the proposed approach. Figure 4(a) gives the damage measures with target first excursion probability for the initial and final structures. As can be seen, the initial structure does not meet the reliability constraints. Figure 4(a) also shows how the final structures, considering either Method 1 or 2, not only meet the reliability constraints but do so with all constraints in a near active state (i.e. damage measure thresholds with target failure probability near the limiting value of $\tilde{D}=1$ ) and attests to the ability of the proposed method in finding high quality final designs. Figure 4(b) shows the performance of the initial and final structures in terms of the original first excursion constraints and, as expected, shows the perfect equivalence between the original and inverse constraint formulations. Because for the optimal structures the failure probabilities associated with the reliability constraints converge to the target probability $\mathrm{P}_{0_{j}}$, the coefficient of variation of the estimated failure probabilities can be simply estimated as [26]:

$$
\delta_{\mathrm{P}\left(G_{j} \leq 0\right)}=\sqrt{\frac{1-\mathrm{P}_{0_{j}}}{N_{s} \mathrm{P}_{0_{j}}}}=0.115
$$

where $N_{s}=75000$ and $\mathrm{P}_{0_{j}}=0.001$. This convergence property can be used to estimate, a priori, the number of samples to be used in the Monte Carlo simulation of each design cycle for achieving a desired level of accuracy in the estimate of the performance of the system.

The maximum first excursion probability of the threshold $\tilde{D}=1$ over all constraints is shown for each design cycle in Fig. 5. It is apparent that the design history for Method 1 is smoother than that seen for Method 2, especially during the later design cycles. Indeed, a small but noticeable oscillation is apparent for the design history relative to Method 2, even after the problem has, for all intents and purposes, converged. This indicates how 


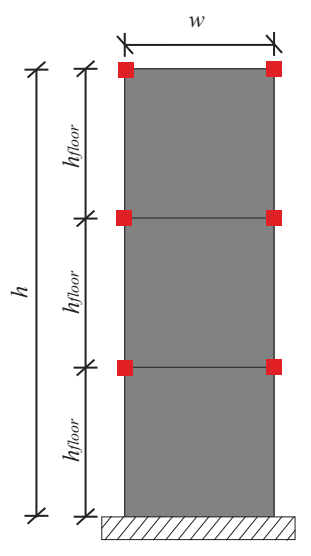

(a)

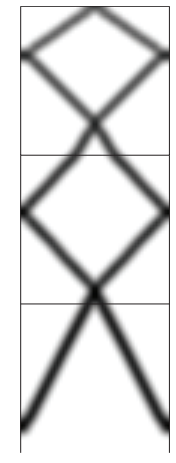

(b)

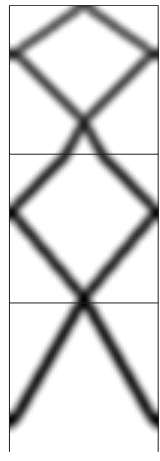

(c)

- Master Nodes

Figure 3: Initial and final topologies for the baseline system of Case Study 1: (a) Initial structure; (b) Final structure considering Method 1; (c) Final structure considering method 2 .

Method 1 will lead to less sample sensitive values of $\tilde{\Upsilon}_{j}$ and consequentially a more stable convergence and therefore would seem preferable over Method 2 .

\subsubsection{Case Study 1: effects of parameter variation}

In this section a parametric study is carried out on the baseline system of Fig. 3(a) with the aim of initially studying: (1) the importance of considering epistemic and system uncertainties; (2) the effects of varying the target failure probability of the constraints; (3) the influence of the secondary system; (4) the comparison of the topology shown in Fig. 3(b) and Fig. 3(c) to a more traditional scheme.

With respect to point (1), Fig. 7(b) shows the optimal structure while considering the mean value of the vector $\mathbf{U}_{s}$ and the median value of the vector $\boldsymbol{\epsilon}$. The system is therefore subject exclusively to uncertainties in the ground motions, i.e. the stochastic excitation generated by the white noise sequence $\mathbf{X}$, and event intensity through the parameters $M$ and $r$ which take on the distributions of Section 5.1. As can be seen from Fig. 7(b), the final topology has changed significantly as compared to the topology of Fig. 7(a) where all sources of uncertainty were considered. In particular, from Fig. 8, it can be seen that the system is around $50 \%$ lighter, as compared to the baseline system, which is an indication of the overall reduction in load that has 


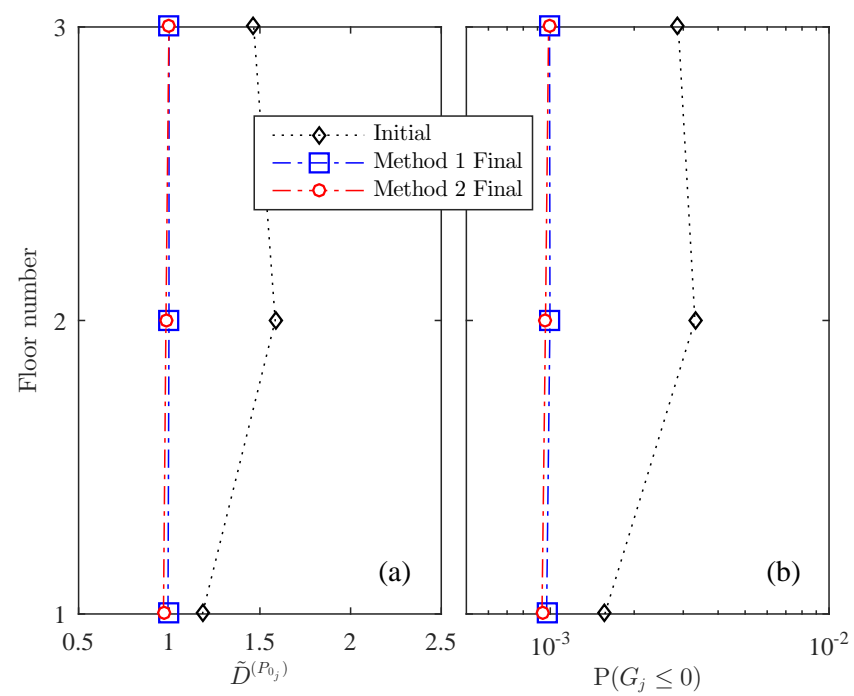

Figure 4: Baseline system of Case Study 1: (a) Damage measure thresholds with target first excursion probability at each floor for the initial and final structures; (b) Original first excursion constraints (Eq. (10)) at each floor for the initial and final structures.

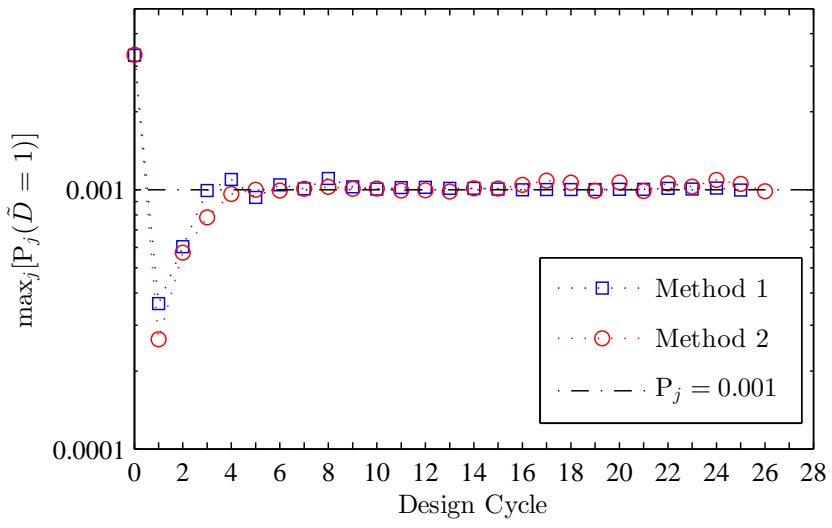

Figure 5: Baseline system of Case Study 1: Maximum failure probability for $\tilde{D}=1$ over all constraints for each design cycle. 


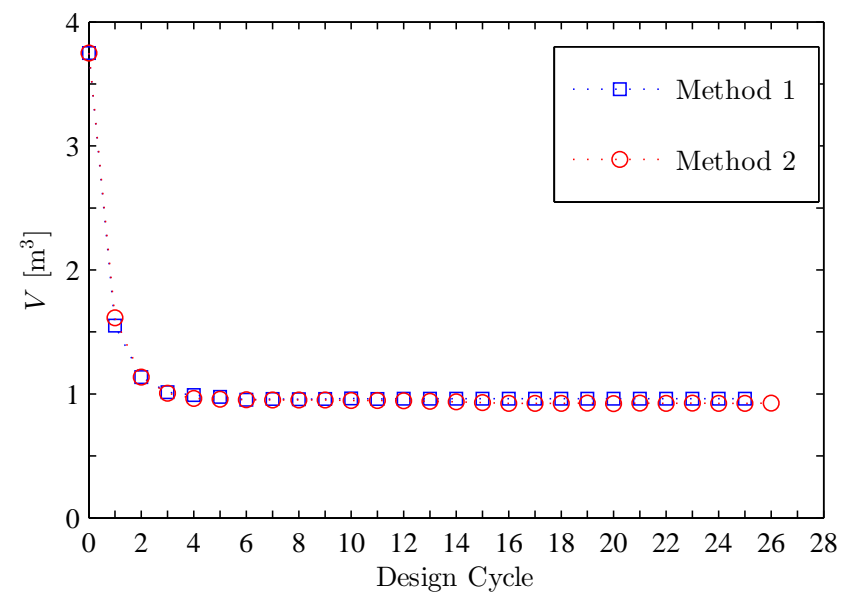

Figure 6: Baseline system of Case Study 1: Volume histories.

occurred as a result of ignoring the epistemic and system uncertainties. This simple example clearly illustrates the importance of considering all sources of uncertainty if optimal topologies are to be defined. With respect to the performance of the proposed approach while considering only uncertainty in the loading, Figs. 8 to 10 show analogous convergence properties as seen for the baseline system.

In consideration of point (2), Fig. 7(c) shows the final structure obtained considering a target failure probability for the reliability constraints of $\mathrm{P}_{0_{j}}=0.01$. As would be expected and shown in Fig. 8, the resulting system is considerably lighter than the baseline system for which a target failure probability of $\mathrm{P}_{0_{j}}=0.001$ was considered. It is interesting to observe the similarities, in terms of the final weight and topology, between the structure obtained considering exclusively loading uncertainty (Fig. 7(b)) and that obtained considering $\mathrm{P}_{0_{j}}=0.01$ (Fig. $7(\mathrm{c})$ ). Indeed, the neglect of system and epistemic uncertainties will in general result in a significant underestimate of the failure probability which can lead to noticeable changes in the final designs identified through topology optimization (e.g. the structural system of Fig. 7(b) instead of that of 7(a)). Figures 8 to 10 show the insensitivity of the proposed approach for variations in the target failure probability with convergence properties all but the same as the baseline system of Section 5.3.1.

With respect to point (3), Fig. 11(a) and Fig. 11(b) show the comparison 


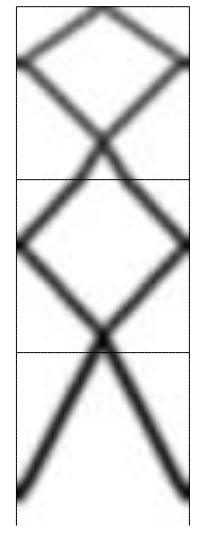

(a)

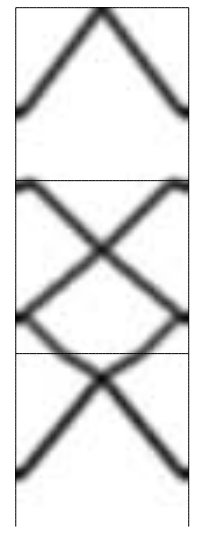

(b)

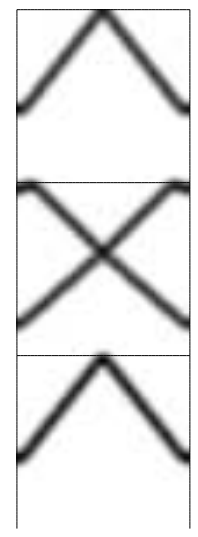

(c)

Figure 7: Case Study 1: (a) Final topology of the baseline system; (b) Final topology considering only load uncertainties; (c) Final topology for baseline system with increased target failure probabilities.

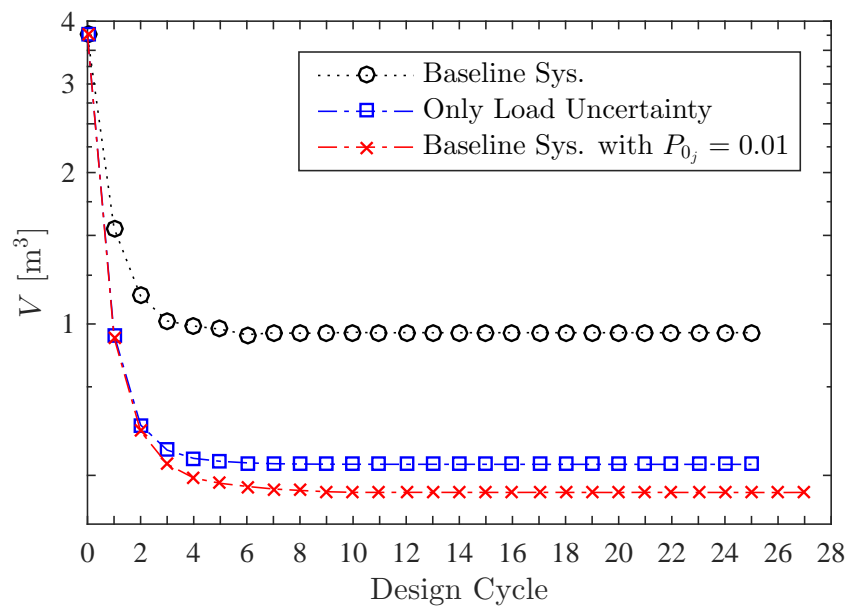

Figure 8: Case Study 1: Volume histories for the system with only load uncertainties or increased target failure probabilities. 


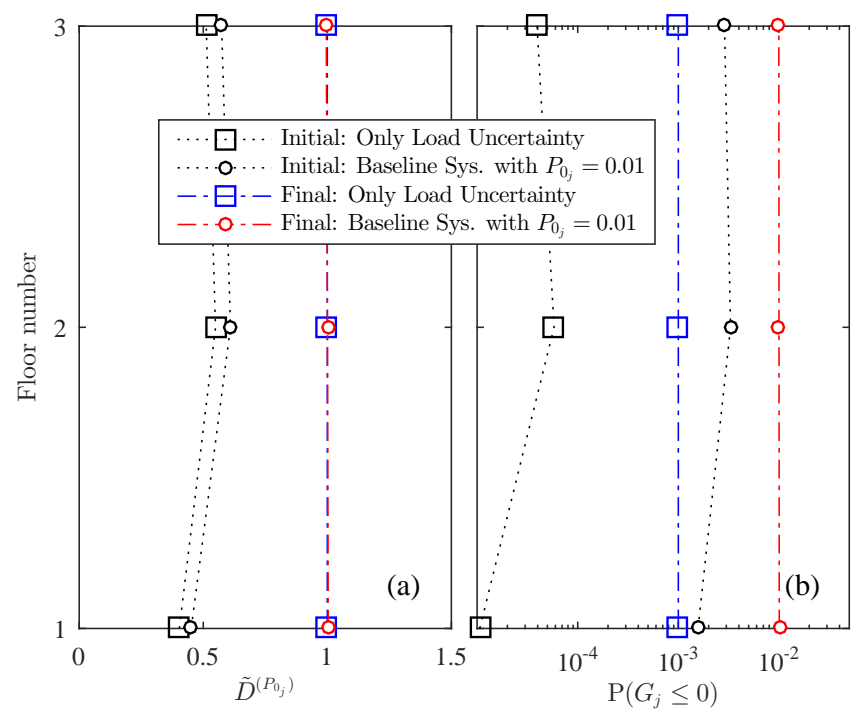

Figure 9: Case Study 1: (a) Damage measure thresholds with target first excursion probability at each floor for the initial and final structures of the system with only load uncertainties or increased target failure probabilities; (b) Original first excursion constraints (Eq. (10)) at each floor for the initial and final structures of the system with with only load uncertainties or increased target failure probabilities.

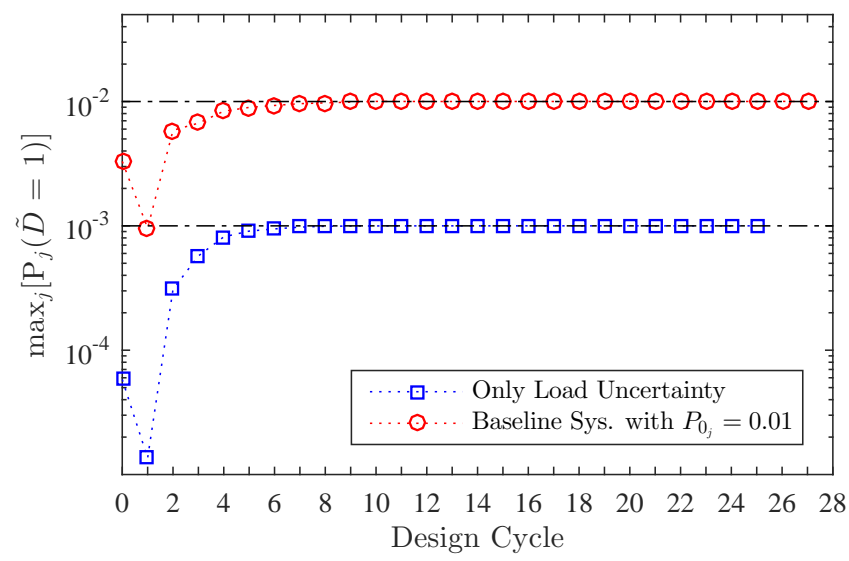

Figure 10: Case Study 1: Maximum failure probability for $\tilde{D}=1$ over all constraints for each design cycle of the system with only load uncertainties or increased target failure probabilities. 
between the baseline topology of Section 5.3.1 and that obtained while considering the Young's modulus of the steel of the secondary system reduced by $90 \%$. It is evident from Fig. 11(b) that the reduction in the stiffness of the secondary system has caused a more complete structural system to appear with both bracing and column elements now defining the lateral load-resisting system. As shown in Fig. 12, the increase in material volume is around $50 \%$ compared to the baseline structure with full secondary system. Figures 12 to 14 show how the convergence properties of the proposed framework is not affected by the reduction secondary system stiffness.

Finally, focusing on point (4), Fig. 11(c) shows the topology obtained by considering the design domain and secondary system connected only at the Master Nodes (Fig. 3(a)). This modeling change effectively ensures that the final topology will pass through the Master Nodes as this now represents the only load path between the secondary system and the lateral load-resisting system. The result of this on the final topology is the identification of a more traditional X-bracing solution, as shown in Fig. 11(c). This is an interesting result as it shows the versatility of topology optimization, and in particular the proposed framework, for obtaining solutions where designability may be an issue. For instance, for the design problem considered in this case study, it may be more desirable from a design standpoint to have the connection points of the bracing and columns aligned to each floor. It is also interesting to compare the final material volume of the X-brace structure of Fig. 11(c) with the baseline structure of Fig. 11(a). Indeed, at first glance, it may not be immediately obvious way the structure of Fig. 11(a)-for which the joints of the secondary system and lateral load-resisting system do not match-is found for the more general complete system (i.e. frame elements of the secondary system translationally connected to all intersecting nodes of the shell elements of the design domain). However, as shown in Fig. 12, the X-brace solution is around 10\% heavier than the baseline solution of Section 5.3.1 and is therefore a sub-optimal solution. Figures 12 to 14 once again show the robustness of the proposed approach in terms of convergence properties as the complete system is changed in order to guide to final design towards desired outcomes.

\subsection{Case Study 2: mid-rise building example}

The second case study considers the topology design of the lateral loadresisting system of a 10-story mid-rise building structure. The frame has 2 bays, each of which has a width of $8 \mathrm{~m}$ while the floor-to-floor height is 


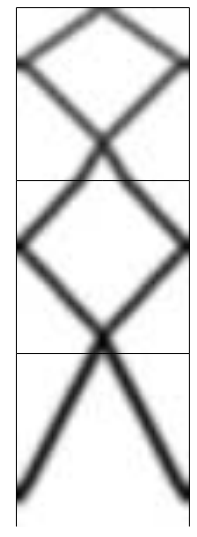

(a)

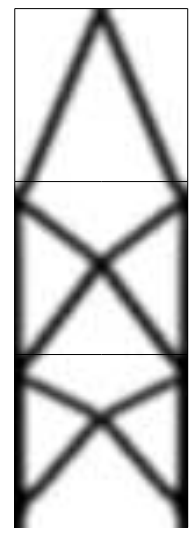

(b)

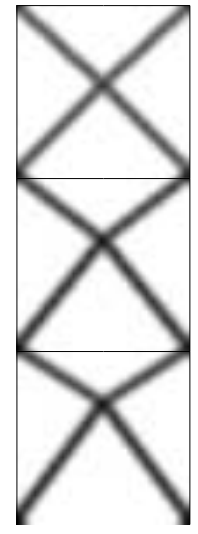

(c)

Figure 11: Case Study 1: (a) Final topology of the baseline system; (b) Final topology considering a $90 \%$ reduction in the Young's modulus of the secondary system's steel; (c) $\mathrm{X}$-brace topology obtained through modification of the complete system.

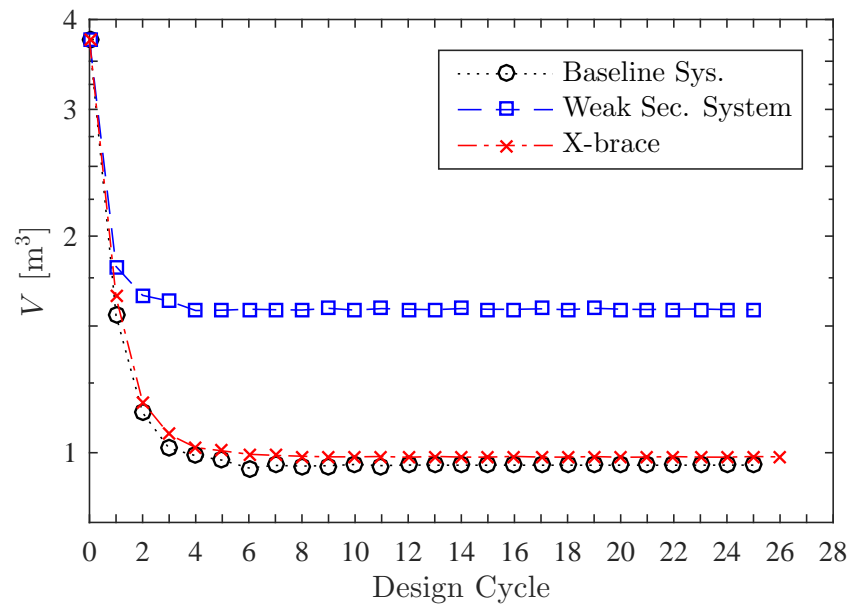

Figure 12: Case Study 1: Volume histories for the system with weak secondary system or imposed X-brace solution. 


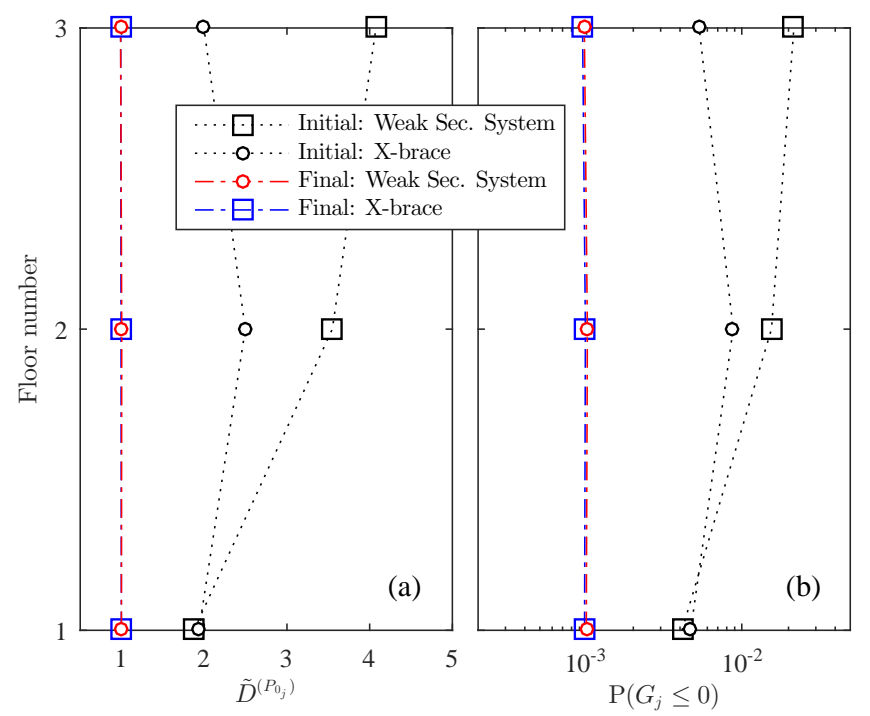

Figure 13: Case Study 1: (a) Damage measure thresholds with target first excursion probability at each floor for systems with weak secondary system or imposed X-brace solution; (b) Original first excursion constraints (Eq. (10)) at each floor for the system with weak secondary system or imposed X-brace solution.

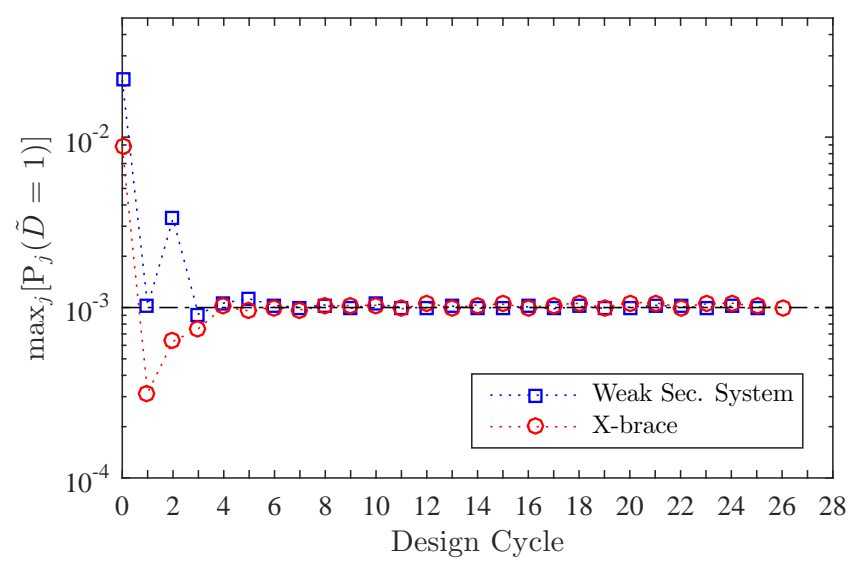

Figure 14: Case Study 1: Maximum failure probability for $\tilde{D}=1$ over all constraints for each design cycle for the system with weak secondary system or imposed X-brace solution. 
$4 \mathrm{~m}(w=16 \mathrm{~m}, h=40 \mathrm{~m})$. Reliability constraints are considered for all 10 drift responses. For each constraint the critical threshold was taken as $\tilde{D}=1$. The initial design domain is indicated by the gray area of Fig. 15(a). The design domain is modeled using 10240 Q4 elements with dimensions $0.25 \mathrm{~m} \times 0.25 \mathrm{~m}$ and a thickness of $0.5 \mathrm{~m}$. Symmetry was imposed about the vertical centerline therefore resulting in a total of 5120 design variables. The planar elements were given the material properties of reinforced concrete with Young's modulus equal to $21 \mathrm{GPa}$. A linear density filter with a radius of $r_{\text {min }}=0.75 \mathrm{~m}$ was used. For the secondary system, W8 $\times 21$ standard steel members where considered for both beams and columns. These were modeled as in Case Study 1 through a series of beam elements of length $0.25 \mathrm{~m}$ (i.e. equal to the side dimensions of the Q4 elements) translationally connected at each end to the quadrilateral mesh for a total of 672 beam elements. Master nodes were considered at all the intersections of the beams and columns of the secondary system therefore resulting in three master nodes at each floor for a total of 30 nodes. A nonstructural carried mass was also considered the entity of which was calculated considering a mass density of $140 \mathrm{~kg} / \mathrm{m}^{3}$ with an out-of-plane influence width of $8 \mathrm{~m}$. Method 1 (Section 4.1) was used to define $\tilde{\Upsilon}_{j}$ due to the superior convergence properties demonstrated in the previous case study.

As opposed to Case Study 1, in this case study a move limit was imposed on the independent design variable vector $\mathbf{y}$ (related to the normalized material design design variable vector $\boldsymbol{\rho}$ through the linear filter $\varphi_{e}$ ) with the aim of improving convergence smoothness of the reliability constraints in the first few design cycles. Indeed, from Figs. 5, 10 and 14 it can be seen that the maximum failure probability over all constraints can vary quite significantly in the first few design cycles. By imposing a move limit on $\mathbf{y}$ before the resolution of each sub-problem, $\boldsymbol{\Psi}_{j}$ will be updated more frequently during the early stages of the design process therefore ensuring a more steady convergence of the reliability constraints. In particular, for all the following examples a move limit of \pm 0.2 around the current design point $\mathbf{y}_{0}$ was considered. This implies that each sub-problem is solved in the interval $\left[\max \left(\varepsilon, \mathbf{y}_{0}-0.2\right), \min \left(1, \mathbf{y}_{0}+0.2\right)\right]$ with $\varepsilon$ a small number, taken here equal to 0.001, necessary for avoiding numerical instabilities.

\subsubsection{Case Study 2: baseline structure}

The baseline system for Case Study 2 was defined considering the uncertainties reported in Sections 5.1 and 5.2. The target first excursion proba- 


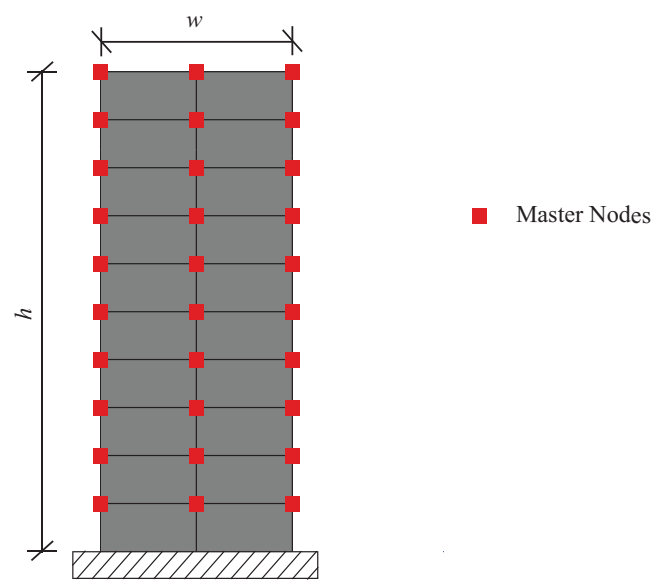

Figure 15: Schematic of the complete system of Case Study 2.

bilities in $[0, T]$ were taken as $\mathrm{P}_{0_{j}}=0.01$ while 50000 samples were used in the Monte Carlo simulation leading to a coefficient of variation in the failure probability estimates of 0.04 . In order to study the effects of modal truncation on the final topologies, the proposed framework was run considering $1,2,3$ and 4 vibration modes in the definition of the load-effect model of Section 3.2.1 (i.e. $k=1,2,3$ and 4) for a total of $78.37 \%, 88.40 \% 93.37 \%$ and $98.73 \%$ of total participating mass respectively. The final topologies are shown in Fig. 16 where the effect of mode truncation on the final topologies is clearly seen. Indeed, as the number of vibration modes increases, the number of bracing elements also increases in order to provide adequate stiffness against the higher modes that, due to the seismic excitation and low damping of the system, contribute significantly to the response of the structure. At this juncture it should be observed that the inclusion of higher modes has a negligible effect on the computational efficiency of the proposed approach as they are estimated in the reduced system of Section 3.3.1. Therefore, within the proposed framework, the user is free to include as many modes as deemed adequate for describing the response of the system. Figure 17 shows the material volume convergence histories for the structures shown in Fig. 16 where the increasing weight of the final system as vibration modes are added is clearly evident. The smoothness of convergence seen in the material volume, i.e. the objective function, is an indication of the quality of approximations assumed during the derivation of the sub-problem of Section 4.2. Figure 18 


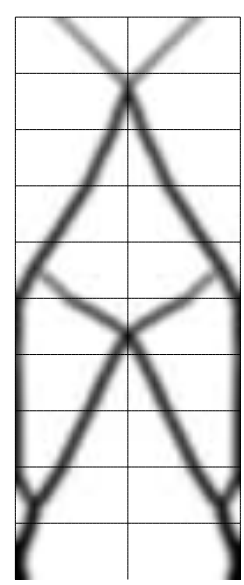

(a)

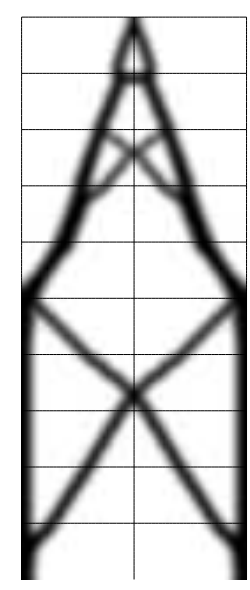

(b)

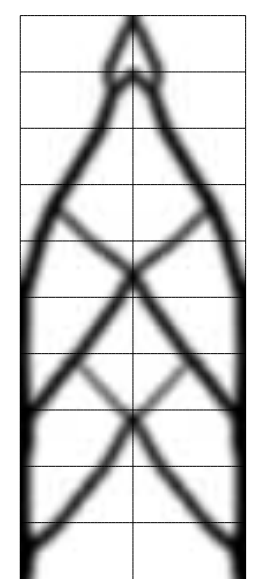

(c)

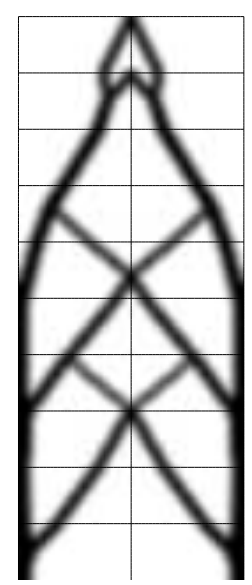

(d)

Figure 16: Baseline system of Case Study 2: (a) Final structure considering only the first vibration mode; (b) Final structure considering the first two vibration modes; (c) Final structure considering three vibration modes; (d) Final structure considering four vibration modes.

shows the initial and final constraint values for each system in terms of both the damage measures with target first excursion probability, Fig. 18(a), as well as the original constraint formulation, Fig. 18(b), of Eq. (10). The number of near active constraints (i.e. constraints at their limiting value) at the end of the optimization is once again a clear indication of the potential of the proposed approach. The perfect equivalence between the original first excursion constraints (Fig. 18(b)) and the inverse constraints (Fig. 18(a)) is again evident with both formulations at their limiting values by the end of the optimization process. Finally, Fig. 19 shows the convergence history of the maximum first excursion probability over all constraints. This figure clearly shows the ability of the proposed method in rapidly finding solutions that meet the constraints. Indeed, it can be seen that structures that practically satisfy the original constraints are being generated after only a dozen or so design cycles (Monte Carlo simulations). The greater smoothness of the convergence seen for all 4 cases as compared to those of Case Study 1 indicates the beneficial effects of considering a move limit on independent design variable vector $\mathbf{y}$. Indeed, strictly speaking, the proposed approach is based on a local approximation of the reliability constraints. 


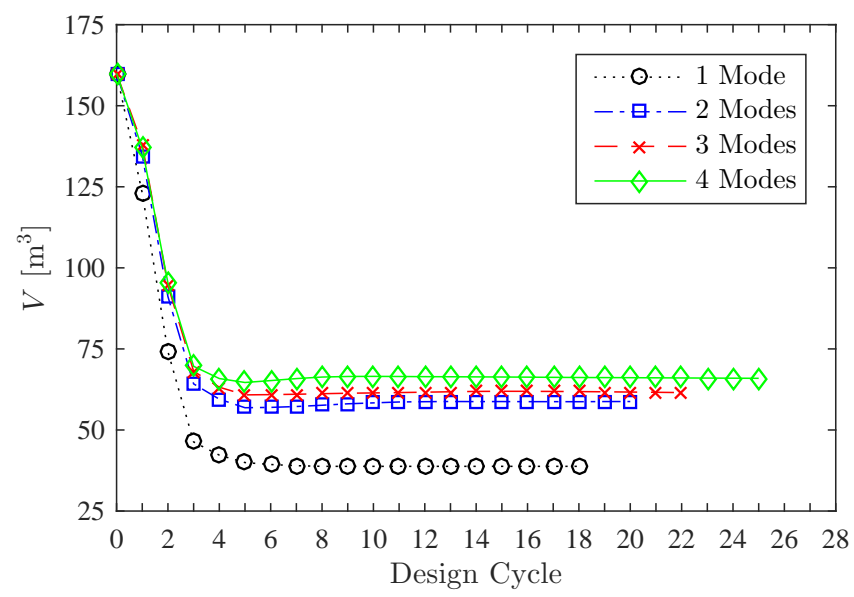

Figure 17: Baseline system of Case Study 2: Volume histories.

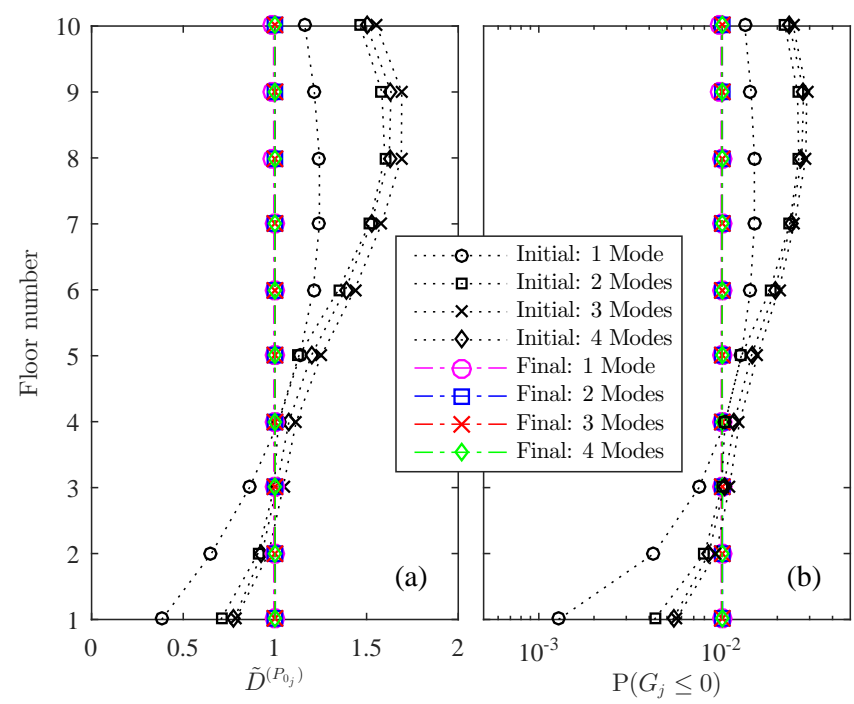

Figure 18: Baseline system of Case Study 2: (a) Damage measure thresholds with target first excursion probability at each floor for the initial and final structures; (b) Original first excursion constraints (Eq. (10)) at each floor for the initial and final structures. 


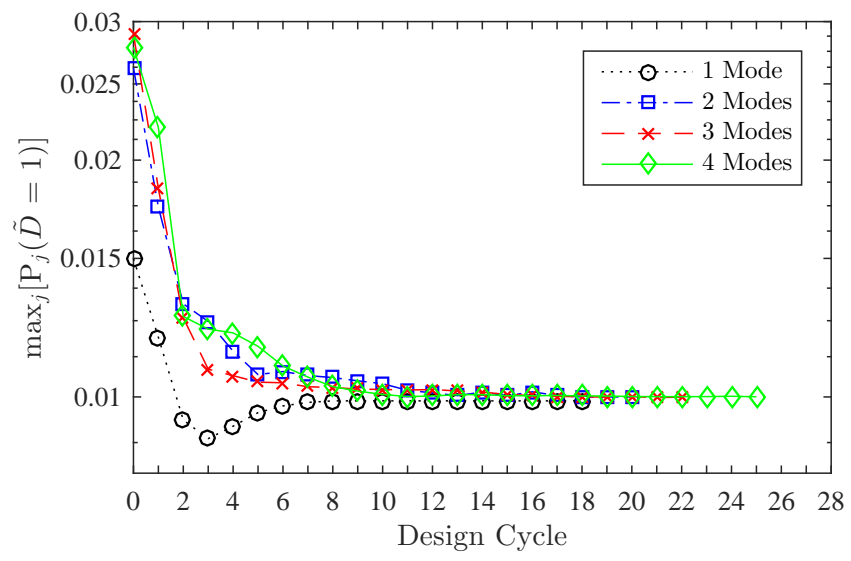

Figure 19: Baseline system of Case Study 2: Maximum failure probability for $\tilde{D}=1$ over all constraints for each design cycle.

\subsubsection{Case Study 2: effects of parameter variation}

The aim of this section is to initially investigate the effects of changing the failure probability of the constraints as well as the stability of the proposed framework when considering exclusively stochastic excitation. In this respect, Fig. 20(a) shows the topology obtained considering the baseline system of Section 5.4.1 with 4 vibration modes and, however, a target failure of $\mathrm{P}_{0_{j}}=0.005$. The coefficient of variation of the estimated failure probabilities is 0.063 as 50000 samples were again considered during the Monte Carlo simulations. From Fig. 20(a) it can be seen that, compared to the structure obtained for a target failure probability of $\mathrm{P}_{0_{j}}=0.01$ (Fig. 16(d)), the system obtained with $\mathrm{P}_{0_{j}}=0.005$ is generally heavier and more braced. This is in line with engineering intuition as a lowering of the failure probability of the system will in general require a stiffer system. Figures 21 to 23 show how the overall behavior of the proposed framework is not affected by a variation in the target failure probability. Indeed, Fig. 22 shows how the reliability constraints are once again strictly met at the end of the optimization process while Figs. 21 and 23 show analogous convergence properties as before for the objective and constraint functions.

Figure 20(b) shows the topology obtained while considering the mean value of the vector $\mathbf{U}_{s}$ and the median value of the vector $\boldsymbol{\epsilon}$. In particular, in contrast to the system studied in Section 5.3.2, here the moment magnitude, $M$, and the fault plane distance, $r$, are fixed at 7.5 and $30 \mathrm{~km}$ respectively. The system is therefore excited exclusively by the non-stationary stochastic 


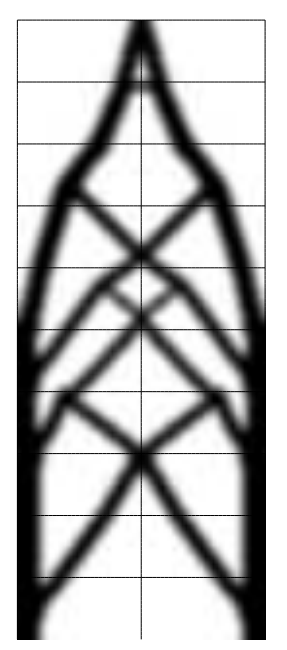

(a)

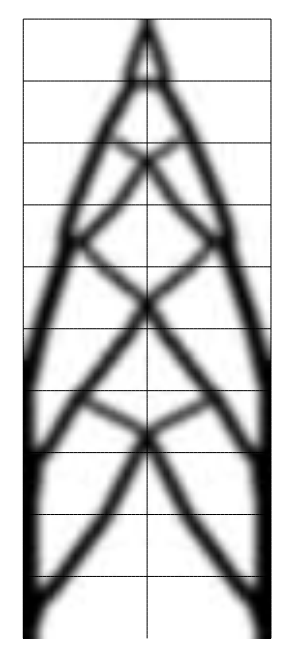

(b)

Figure 20: Case Study 2: (a) Final topology for system with reduced target failure probabilities; (b) Final topology considering only stochastic excitation.

excitation generated by the white noise sequence $\mathbf{X}$. A target failure probability of $\mathrm{P}_{0_{j}}=0.002$ was considered while 50000 samples were used in the Monte Carlo simulation for a coefficient of variation in the failure probability estimates of 0.1. Four vibration modes were considered in the response analysis for a total of $99 \%$ of participating mass. While the topology obtained under these circumstances shows similarities to those obtained considering all sources of uncertainty, the importance of considering a full range of uncertainties during topology optimization of dynamic buildings systems subject to earthquake excitation would seem evident. Indeed, compared to the systems of Figs. 16(d) and 20(a), the upper portion of the system shown in Fig. 20 (b) is characterized by an extra X-brace as well as a generally narrower appearance. Figures 21 to 23 show how the overall behavior of the proposed framework is in no way affected by considering exclusively stochastic excitation with convergence properties for the objective and constraint functions remaining unaltered with respect to the other cases investigated in work.

\subsection{Comments}

This section studied the convergence properties of the proposed approach for non-stationary stochastic earthquake excitation. This represents arguable one of the more complex and general scenarios under which to optimize, as 


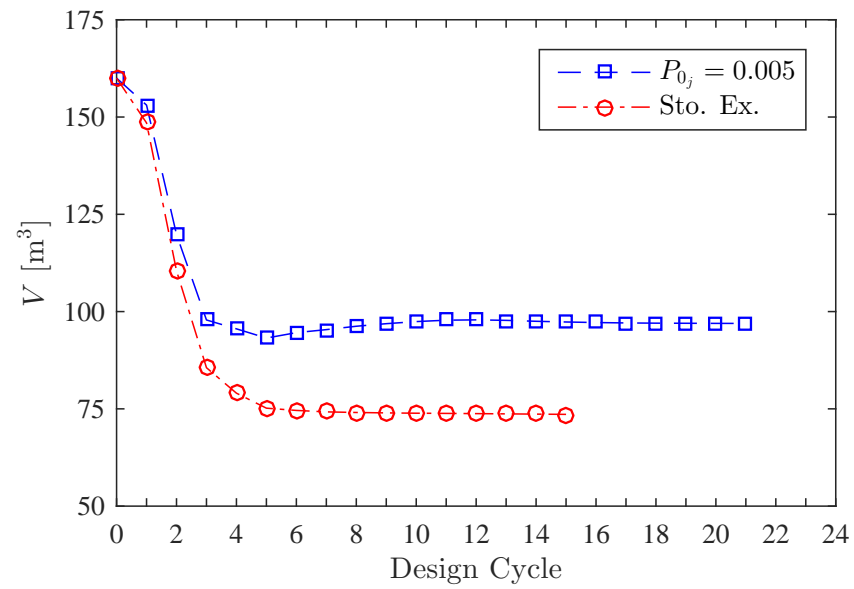

Figure 21: Case Study 2: Volume histories for the system with reduced target failure probability or only stochastic excitation.

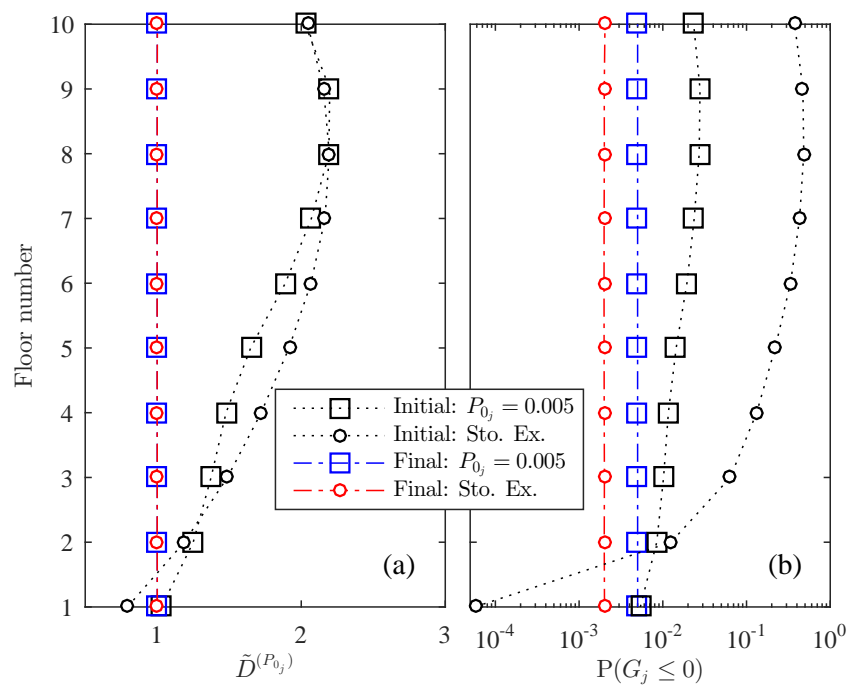

Figure 22: Case Study 2: (a) Damage measure thresholds with target first excursion probability at each floor for the initial and final structures of the system with reduced target failure probability or only stochastic excitation; (b) Original first excursion constraints (Eq. (10)) at each floor for the initial and final structures of the system with reduced target failure probability or only stochastic excitation. 


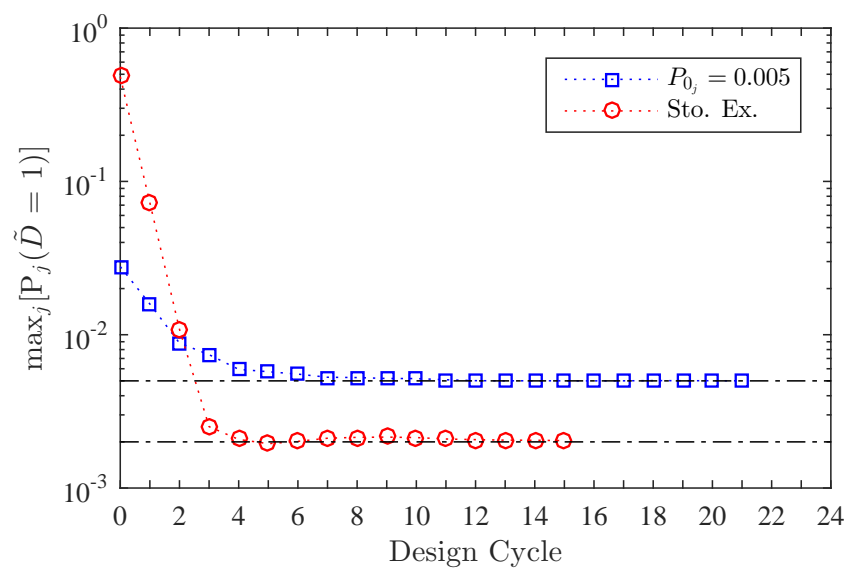

Figure 23: Case Study 2: Maximum failure probability for $\tilde{D}=1$ over all constraints for each design cycle of the system with reduced target failure probability or only stochastic excitation.

the dependency of the forcing functions, $f$, on the mass matrix couples $f$ with the design variable vector $\boldsymbol{\rho}$, while the non-statioanrity of $\ddot{z}_{g}$ significantly complicates the treatment of the reliability constraints. Indeed, for stationary excitation the instantaneous failure probability is easily related to the first excursion probability in $[0, T]$ therefore significantly reducing the size of the optimization problem, i.e. the optimization problem can be essentially posed in terms of probabilistic constraints written for an arbitrary instant belonging to $[0, T]$. For non-stationary excitation this is no longer possible and all instants (i.e. the discrete time steps of each realization of f) must be considered. The proposed approach overcomes this significant computational challenge through the definition of the vector $\tilde{\Upsilon}_{j}$ (Section 4.1) which allows non-stationary excitation to be treated without any added complication over stationary excitation. It should also be observed that, because the proposed approach is purely simulation-based, the inclusion of any non-Gaussian features in the excitation is straightforward and would not affect the framework. These properties highlight the strong potential of the proposed approach for solving RBTO problems under a wide variety of potential design scenarios involving stationary/non-stationary Gaussian or non-Gaussian excitation. Finally, in order to illustrate the advantages of considering a framework of this type in seismic design, it is interesting to compare the baseline design obtained from Case Study 2 (reported in Fig. 24(a) for convenience), to a more traditional X-brace designed under the 


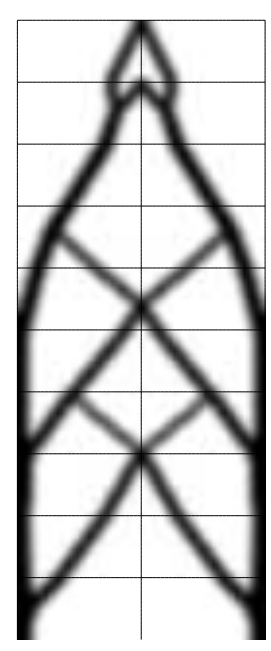

(a)

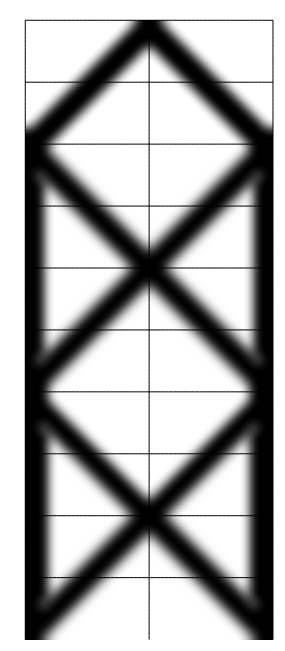

(b)

Figure 24: Comparison between the baseline solution of Case Study 2 and a traditional X-brace solution: (a) Baseline solution of Case Study 2; (b) Traditional X-brace solution.

same loading conditions and uncertainties. Indeed, if the X-brace scheme illustrated is Fig. 24(b) is designed in order to provide the same performance as the system of Fig. 24(a), i.e. maximum failure probability for each reliability constraint less than or equal to $\mathrm{P}_{0_{j}}=0.01$-as shown in Fig. 25-, then a system is obtained that is over $75 \%$ heavier than the system of Fig. 24(a). Indeed, the dependence of seismic loads on the mass of the system together with the role of higher modes will result in lightweight systems that are stiff in all participating modes becoming more highly performing.

\section{Conclusions}

This paper presented a framework for the reliability-based topology optimization of dynamically sensitive uncertain building systems subject to stochastic excitation. In particular, the method is based on describing the reliability of the system through a number of constraints written in terms of first excursion probabilities posed on the generally non-stationary system response. At the core of the method is the definition of an approximate optimization sub-problem that can be entirely defined from the results of a single augmented simulation-based probabilistic analysis carried out with the sole aim of evaluating the reliability constraints. Indeed, no global/local explo- 


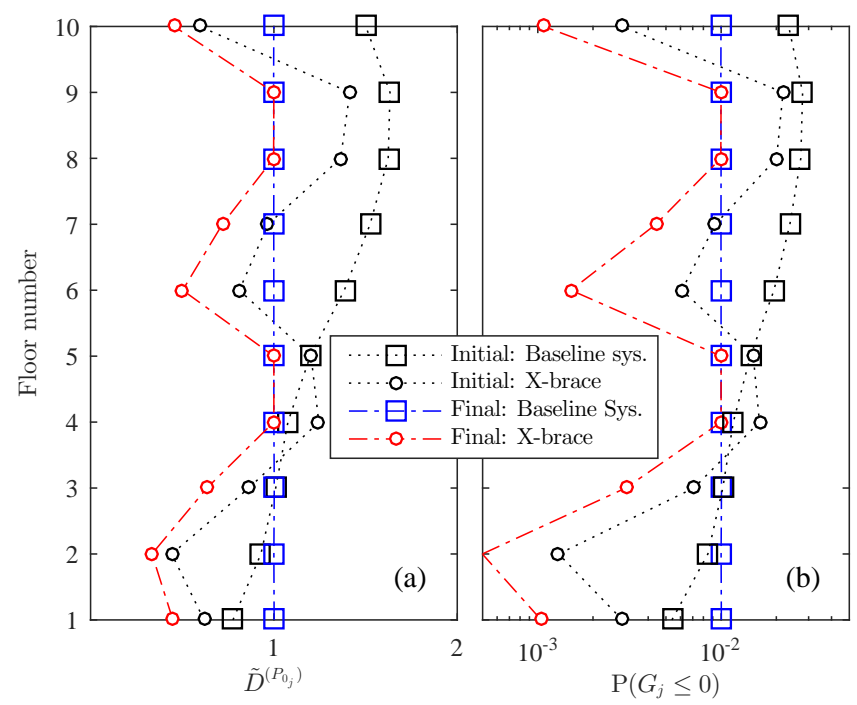

Figure 25: Comparison between the baseline solution of Case Study 2 and a traditional X-brace solution: (a) Damage measure thresholds with target first excursion probability at each floor for the initial and final structures; (b) Original first excursion constraints (Eq. (10)) at each floor for the initial and final structures.

ration of the design space, or sensitivities with respect to the design variables, are necessary for the definition of the sub-problem. This makes the approach practically independent of the size of the design variable vector and therefore perfectly posed for solving topology optimization problems where design variable vectors are typically in the order of thousands. By formulating and solving a limited sequence of sub-problems, a solution is found to the original problem that rigorously meets the original first excursion constraints without the need to impose restrictions on the type of external excitation. The proposed approach is particularly efficient as not only is a limited number of sub-problems formulations necessary before overall convergence, but also the sub-problem takes the form of a classic deterministic and static topology optimization problem which can therefore be solved extremely efficiently. The applicability and strong convergence properties of the proposed approach were illustrated on two case studies that focused on the topology optimization of the lateral load-resisting system of frames subject to non-stationary stochastic earthquake excitation. 


\section{Acknowledgments}

Support for this work was in part provided by the NSF Grant No. CMMI1301008. The authors also would like to thank Krister Svanberg for providing the MMA algorithm, which was used as the optimization algorithm in this research.

\section{Appendix A. Seismic excitation model}

\section{Appendix A.1. The amplitude spectrum}

For the simulation of ground motions, it is typical to describe the amplitude spectrum as the product of the source spectrum $E\left(f ; \mathbf{u}_{g}\right)$, path effect $P\left(f ; \mathbf{u}_{g}\right)$, and site effect $G\left(f ; \mathbf{u}_{g}\right)$ as follows [31]:

$$
A\left(f ; \mathbf{u}_{g}\right)=(2 \pi f)^{2} E\left(f ; \mathbf{u}_{g}\right) P\left(f ; \mathbf{u}_{g}\right) G\left(f ; \mathbf{u}_{g}\right)
$$

The source spectrum is given by:

$$
E\left(f ; \mathbf{u}_{g}\right)=c M_{w} S\left(f ; \mathbf{u}_{g}\right)
$$

where $c$ is a constant and is given by:

$$
c=10^{-20} R_{\Phi} V F /\left(4 \pi R_{0} \rho_{s} \beta_{s}^{3}\right)
$$

where $R_{\Phi}$ is the radiation pattern and is usually averaged over an appropriate range of azimuths and take-off angles, $V=1 / \sqrt{2}$ represents the partition of total shear-wave velocity into horizontal components, $F$ is the free surface amplification and is taken to be 2 in most cases, $\rho_{s}$ and $\beta_{s}$ are the density and shear-wave velocity in the vicinity of the source, and $R_{0}$ is a reference distance and is usually set to $1 \mathrm{~km}$. The term $M_{w}$ in Eq. (A.2) is the seismic moment and is related to the moment magnitude $M$ by the following relationship:

$$
\log _{10} M_{w}=1.5(M+10.7)
$$

and $S\left(f ; \mathbf{u}_{g}\right)$ is the displacement source spectrum. The two-corner pointsource displacement source spectrum developed by Atkinson and Silva [45] for the region of California is adopted for this research:

$$
S\left(f ; \mathbf{u}_{g}\right)=\frac{1-e}{1+\left(f / f_{a}\right)^{2}}+\frac{e}{1+\left(f / f_{b}\right)^{2}}
$$


where the lower corner frequency $f_{a}$, higher corner frequency $f_{b}$, and weighting parameter $e$ are related to the moment magnitude, respectively, by:

$$
\begin{gathered}
\log _{10} f_{a}=2.181-0.496 M \\
\log _{10} f_{b}=2.41-0.408 M \\
\log _{10} e=0.605-0.255 M
\end{gathered}
$$

A simplified path effect may be defined as the multiplication of the geometrical spreading function $Z\left(R_{r}\right)$ and seismic attenuation [31] as follows:

$$
P\left(f ; \mathbf{u}_{g}\right)=Z\left(R_{r}\right) \exp \left[\frac{-\pi f R_{r}}{Q(f) c_{Q}}\right]
$$

where $Q(f)$ is a regional attenuation function, $c_{Q}$ is the seismic velocity used to determine $Q(f)$, and $R_{r}=\sqrt{h^{2}+r^{2}}$ is the radial distance from the earthquake source to the site, where $r$ is the closest distance to the fault plane and $h$ is a moment-dependent "equivalent point-source depth" [45] and is represented by:

$$
\log _{10} h=-0.05+0.15 M
$$

The geometrical spreading function, $Z(R)$, may be given by a piece-wise continuous series of straight line segments as follows:

$$
Z(R)= \begin{cases}\frac{R_{0}}{R} & R \leq R_{1} \\ Z\left(R_{1}\right)\left(\frac{R_{1}}{R}\right)^{p_{1}} & R_{1} \leq R \leq R_{2} \\ \vdots & \\ Z\left(R_{n}\right)\left(\frac{R_{n}}{R}\right)^{p_{n}} & R_{n} \leq R\end{cases}
$$

The site effect may be given as the product of the amplification function $A_{m}(f)$ and the diminution/attenuation $D\left(f ; \mathbf{u}_{g}\right)$ as follows:

$$
G\left(f ; \mathbf{u}_{g}\right)=D\left(f ; \mathbf{u}_{g}\right) A_{m}(f)
$$

The amplification may be described using the empirical curves given in Boore and Joyner [46]. The diminution is used to account for the path-independent loss of high frequency in the ground motions. This effect may be accounted for using the $\kappa_{0}$ filter:

$$
D\left(f ; \mathbf{u}_{g}\right)=\exp \left[-\pi \kappa_{0} f\right]
$$


or the $f_{\max }$ filter:

$$
D\left(f ; \mathbf{u}_{g}\right)=\left(1+\left(\frac{f}{f_{\max }}\right)^{8}\right)^{-1 / 2}
$$

A combination of both filters may also be used [31].

\section{Appendix A.2. Time Envelope}

The temporal characteristics of the excitation are incorporated using the following envelope function:

$$
e_{t}\left(t ; \mathbf{u}_{g}\right)=a_{t}\left(\frac{t}{t_{n}}\right)^{b_{t}} \exp \left[-\frac{c_{t} t}{t_{n}}\right]
$$

where the parameters $a_{t}, b_{t}$, and $c_{t}$ are chosen so that the peak value of the envelope function is equal to unity when time $t=\lambda_{t} t_{n}$ and $e_{t}(t ; M, r)=\eta_{t}$ when $t=t_{n}$. The parameters $a_{t}, b_{t}$, and $c_{t}$ are given by:

$$
\begin{gathered}
b_{t}=\frac{-\lambda_{t} \ln \left[\eta_{t}\right]}{1+\lambda_{t}\left(\ln \left[\lambda_{t}\right]-1\right)} \\
c_{t}=\frac{b_{t}}{\lambda_{t}} \\
a_{t}=\left(\frac{\exp [1]}{\lambda_{t}}\right)^{b_{t}}
\end{gathered}
$$

where $t_{n}=2 T_{w}$ is the time duration parameter and

$$
T_{w}=\frac{1}{2 f_{a}}+0.05 R_{r}
$$

is the duration of strong ground motion $[31,45]$.

\section{Appendix B. Derivation of Eq. (26)}

The threshold $D_{j}^{\left(\mathrm{P}_{j}\right)}$ can be written, without loss of generality, as follows:

$$
D_{j}^{\left(\mathrm{P}_{0_{j}}\right)}(\boldsymbol{\rho})=\bar{D}_{j}(\boldsymbol{\rho})+\gamma_{D_{j}}\left(P_{0_{j}}, \boldsymbol{\rho}\right) \sigma_{D_{j}}(\boldsymbol{\rho})
$$


where $\bar{D}_{j}$ is the mean damage ratio, $\sigma_{D_{j}}$ is the standard deviation of the damage ratio while $\gamma_{D_{j}}$ is the number of standard deviations that the demand threshold $D_{j}^{\left(\mathrm{P}_{0_{j}}\right)}$ is from its mean. The validity of Eq. (21) implies that the following must hold for the mean and standard deviation of $D_{j}[37,38]$ :

$$
\begin{aligned}
\bar{D}_{j}(\boldsymbol{\rho}) & =\frac{\tilde{\boldsymbol{\Gamma}}_{R_{j}}^{T}(\boldsymbol{\rho})}{\bar{C}_{j}} \tilde{\boldsymbol{\psi}}_{j}(\boldsymbol{\rho}) \\
\sigma_{D_{j}}(\boldsymbol{\rho}) & =\frac{\tilde{\boldsymbol{\Gamma}}_{R_{j}}^{T}(\boldsymbol{\rho})}{\bar{C}_{j}}\left(\frac{\mathbf{C}_{\tilde{\boldsymbol{\psi}}_{j}}(\boldsymbol{\rho}) \tilde{\boldsymbol{\Gamma}}_{R_{j}}(\boldsymbol{\rho})}{\sigma_{D_{j}}(\boldsymbol{\rho}) \bar{C}_{j}}\right)
\end{aligned}
$$

where the meaning of the symbols in Eqs. (B.2) are as reported in Section 4.1. By substituting Eqs. (B.2) into Eq. (B.1) the following is obtained:

$$
\begin{aligned}
D_{j}^{\left(\mathrm{P}_{0_{j}}\right)}(\boldsymbol{\rho}) & =\frac{\tilde{\boldsymbol{\Gamma}}_{R_{j}}^{T}(\boldsymbol{\rho})}{\bar{C}_{j}} \tilde{\boldsymbol{\psi}}_{j}(\boldsymbol{\rho})+\gamma_{D_{j}}\left(P_{0_{j}}, \boldsymbol{\rho}\right) \frac{\tilde{\boldsymbol{\Gamma}}_{R_{j}}^{T}(\boldsymbol{\rho})}{\bar{C}_{j}}\left(\frac{\mathbf{C}_{\tilde{\boldsymbol{\psi}}_{j}}(\boldsymbol{\rho}) \tilde{\boldsymbol{\Gamma}}_{R_{j}}(\boldsymbol{\rho})}{\sigma_{D_{j}}(\boldsymbol{\rho}) \bar{C}_{j}}\right) \\
& =\frac{\tilde{\boldsymbol{\Gamma}}_{R_{j}}^{T}(\boldsymbol{\rho})}{\bar{C}_{j}}\left(\overline{\tilde{\boldsymbol{\psi}}}_{j}(\boldsymbol{\rho})+\gamma_{D_{j}}\left(\mathrm{P}_{0_{j}}, \boldsymbol{\rho}\right) \frac{\mathbf{C}_{\tilde{\boldsymbol{\psi}}_{j}}(\boldsymbol{\rho}) \tilde{\boldsymbol{\Gamma}}_{R_{j}}(\boldsymbol{\rho})}{\sigma_{D_{j}}(\boldsymbol{\rho}) \bar{C}_{j}}\right) \\
& =\frac{\tilde{\boldsymbol{\Gamma}}_{R_{j}}^{T}(\boldsymbol{\rho}) \tilde{\boldsymbol{\Psi}}_{j}\left(\mathrm{P}_{0_{j}}, \boldsymbol{\rho}\right)}{\bar{C}_{j}}
\end{aligned}
$$

\section{References}

[1] M. M. Neves, H. Rodrigues, J. M. Guedes, Generalized topology design of structures with a buckling load criterion, Struct. Optimization 10 (1995) 71-78.

[2] A. R. Mijar, C. C. Swan, J. S. Arora, I. Kosaka, Continuum topology optimization for concept design of frame bracing systems, J. Struct. Eng. 124 (1998) 541-550.

[3] Q. Q. Liang, Y. M. Xie, G. P. Steven, Optimal topology design of bracing systems for multistory steel frames, J. Struct. Eng. 126 (2000) $823-829$.

[4] L. L. Stromberg, A. Beghini, W. F. Baker, G. H. Paulino, Application of layout and topology optimization using pattern gradation for the conceptual design of buildings, Struct. Multidisc. Optim. 43 (2011) $165-180$. 
[5] L. L. Stromberg, A. Beghini, W. F. Baker, G. H. Paulino, Topology optimization for braced frames: Combining continuum and beam/column elements, Eng. Struct. 37 (2012) 106-124.

[6] S. Allahdadian, B. Boroomand, A. R. Barekatein, Towards optimal design of bracing system of multi-story structures under harmonic base excitation through a topology optimization scheme, Finite. Elem. Anal. Des. 61 (2012) 60-74.

[7] S. Bobby, S. M. J. Spence, E. Bernardini, D. Wei, A. Kareem, A complete performance-based optimization framework for the design of tall buildings, in: G. Deodatis, B. R. Ellingwood, D. M. Frangopol (Eds.), Safety Reliability, Risk and Life-Cycle Performance of Structures \& Infrastructures (ICOSSAR 2013), CRC Press, 2013.

[8] A. Kareem, S. M. J. Spence, E. Bernardini, S. Bobby, D. Wei, Using computational fluid dynamics to optimize tall building design, CTBUH Journal III (2013) 38-43.

[9] S. Bobby, S. M. J. Spence, E. Bernardini, A. Kareem, A performancebased methodology for the topology design of tall buildings, in: Proc. of Structures Congress 2014, Boston, 2014.

[10] S. Bobby, S. M. J. Spence, E. Bernardini, A. Kareem, Performancebased topology optimization for wind-excited tall buildings: A framework, Eng. Struct. 74 (2014) 242-255.

[11] G. Schuëller, H. A. Jensen, Computational methods in optimization considering uncertainties - an overview, Comput. Methods Appl. Mech. Engrg. 198 (2008) 2-13.

[12] M. A. Valdebenito, G. I. Schuëller, A survey on approaches for reliability-based optimization, Struct. Multidisc. Optim. 42 (2010) 645663.

[13] K. Maute, D. M. Frangopol, Reliability-based design of MEMS mechanisms by topology optimization, Comput. Struct. 81 (2003) 813-824.

[14] H.-S. Jung, S. Cho, Reliability-based topology optimization of geometrically nonlinear structures with loading and material uncertainties, Finite. Elem. Anal. Des. 43 (2004) 311-331. 
[15] M. Tootkaboni, A. Asadpoure, J. K. Guest, Topology optimization of continuum structures under uncertainty-A polynomial chaos approach, Comput. Methods Appl. Mech. Engrg. 201-204 (2012) 263-275.

[16] M. Jalalpour, J. K. Guest, T. Igusa, Reliability-based topology optimization of trusses with stochastic stiffness, Struct. Saf. 43 (2013) $41-49$.

[17] G. Kharmanda, N. Olhoff, A. Mohamed, M. Lemaire, Reliability-based topology optimization, Struct. Multidisc. Optim. 26 (2004) 295-307.

[18] K. Mogami, S. Nishiwaki, K. Izui, M. Yoshimura, N. Kogiso, Reliabilitybased structural optimization of frame structures for multiple failure criteria using topology optimization techniques, Struct. Multidisc. Optim. 32 (2006) 299-311.

[19] J. K. Guest, T. Igusa, Structural optimization under uncertain loads and nodal locations, Comput. Methods Appl. Mech. Engrg. 1 (2008) $116-124$.

[20] M. Silva, D. A. Tortorelli, J. A. Norato, C. Ha, H.-R. Bae, Component and system reliability-based topology optimization using a single-loop method, Struct. Multidisc. Optim. 41 (2010) 87-106.

[21] T. H. Nguyen, J. Song, G. H. Paulino, Single-loop system reliabilitybased topology optimization considering statistical dependence between limit-states, Struct. Multidisc. Optim. 44 (2011) 593-611.

[22] M. Jalalpour, J. K. Guest, T. Igusa, Optimal design of trusses with geometric imperfections: Accounting for global instability, Int. J. Numer. Meth. Engng. 48 (2013) 3011-3019.

[23] J. Chun, J. Song, G. H. Paulino, System reliability based topology optimization of structures under stochastic excitations, in: G. Deodatis, B. R. Ellingwood, D. M. Frangopol (Eds.), Safety Reliability, Risk and Life-Cycle Performance of Structures \& Infrastructures (ICOSSAR 2013), CRC Press, 2013.

[24] J. Chun, J. Song, G. H. Paulino, Structural topology optimization under constraints on instantaneous failure probability, Struct. Multidisc. Optim. 53 (2016) 773-799. 
[25] A. Der Kiureghian, The geometry of random vibrations by FORM and SORM, Prob. Eng. Mech. 15 (2000) 81-90.

[26] P. Pinto, R. Giannini, P. Franchin, Seismic Reliability Analysis of Structures, IUSS Press, Pavia, Italy, 2004.

[27] M. McCullough, D. Kwon, A. Kareem, L. Wang, Efficacy of averaging interval for nonstationary winds, J. Eng. Mech. 140 (2014) 1-19.

[28] A. K. Chopra, Dynamics of Structures - Theory and Applications to Earthquake Engineering, second ed., Prentice Hall, Upper Saddle River, New Jersey, 2001.

[29] B. Bourdin, Filters in topology optimization, Int. J. Numer. Meth. Engng. 50 (2001) 2143-2158.

[30] G. I. Schuëller, H. J. Pradlwarter, P. S. Koutsourelakis, A comparative study of reliability estimation procedures for high dimensions using $\mathrm{FE}$ analysis, in: 16th ASCE Engineering Mechanics Conference, July 16-18, Seattle, Washington, USA., 2003.

[31] D. M. Boore, Simulation of ground motion using the stochastic method, Pure Appl. Geophys. 160 (2003) 635-676.

[32] C. Vetter, A. A. Taflanidis, Global sensitivity analysis for stochastic ground motion modeling in seismic-risk assessment, Soil Dyn. Earthq. Eng. 38 (2012) 128-143.

[33] S. M. J. Spence, A. Kareem, Data-enabled design and optimization (DEDOpt): Tall steel buildings frameworks, Comput. Struct. 134 (2013) $134-147$.

[34] S. M. J. Spence, A. Kareem, Tall buildings and damping: a conceptbased data-driven model, J. Struct. Eng. 140 (2014) 04014005-1-15.

[35] S. M. J. Spence, E. Bernardini, Y. Guo, A. Kareem, M. Gioffrè, Natural frequency coalescing and amplitude dependent damping in the windexcited response of tall buildings, Prob. Eng. Mech. 35 (2014) 108-117.

[36] G. Schuëller, H. Pradlwarter, P. Koutsourelakis, A critical appraisal of reliability estimation procedures for high dimensions, Probab. Eng. Mech. 19 (2004) 463-474. 
[37] X. Chen, A. Kareem, Coupled dynamic analysis and equivalent static wind loads on buildings with three-dimensional modes, J. Struct. Eng. 131 (2005) 1071-1082.

[38] X. Chen, A. Kareem, Dynamic wind effects on buildings with 3D coupled modes: Application of high frequency force balance measurements, J. Eng. Mech. 131 (2005) 1115-1125.

[39] M. Kasperski, Extreme wind load distributions for linear and nonlinear design, Eng. Struct. 14 (1992) 27-34.

[40] K. Svanberg, The method of moving asymptotes - a new method for structural optimization, Int. J. Numer. Meth. Engng. 24 (1987) 359-373.

[41] C. Vetter, A. A. Taflanidis, Comparison of alternative stochastic ground motion models for seismic risk characterization, Soil Dyn. Earthq. Eng. 58 (2014) 48-65.

[42] S. L. Kramer, Geotechnical Earthquake Engineering, Prentice-Hall, Inc., Upper Saddle River, New Jersey, 1996.

[43] M. P. Bendsøe, Optimal shape design as a material distribution problem, Struct. Optimization 1 (1989) 193-202.

[44] G. Rozvany, M. Zhou, T. Birker, Generalized shape optimization without homogenization, Struct. Optimization 4 (1992) 250-252.

[45] G. M. Atkinson, W. Silva, Stochastic modeling of California ground motions, B. Seismol. Soc. Am. 90 (2000) 255-274.

[46] D. M. Boore, W. B. Joyner, Site amplifications for generic rock sites, Bull. Seismol. Soc. Am. 87 (1997) 327-341. 